\title{
The molecular architecture of vimentin filaments
}

2

3 Matthias Eibauer ${ }^{1}$, Miriam S. Weber ${ }^{1}$, Yagmur Turgay $^{1 \$}$, Suganya Sivagurunathan ${ }^{2}$,

4 Robert D. Goldman², and Ohad Medalia ${ }^{1^{*}}$

5

6

${ }^{1}$ Department of Biochemistry, University of Zurich, Winterthurerstrasse 190, 8057 Zurich, Switzerland

2 Department of Cell and Molecular Biology, Northwestern University Feinberg School of Medicine, 303 E Chicago

Avenue, Chicago, Illinois, USA

$\$$ Current address: Department of Health Sciences and Technology, ETH Zurich, Vladimir-Prelog-Weg 1-5/10, 8093 Zurich, Switzerland

*Correspondence: omedalia@bioc.uzh.ch

\section{ABSTRACT}

Intermediate filaments are integral components of the cytoskeleton in metazoan cells. Due to their specific viscoelastic properties they are principal contributors to flexibility and tear strength of cells and tissues. Vimentin, an intermediate filament protein expressed in fibroblasts and endothelial cells, assembles into $\sim 11 \mathrm{~nm}$ thick biopolymers, that are involved in a wide variety of cellular functions in health and disease. Here, we reveal the structure of insitu polymerized vimentin filaments to a subnanometer resolution by applying cryo-electron tomography to mouse embryonic fibroblasts grown on electron microscopy grids. We show that vimentin filaments are tube-like assemblies with a well-defined helical symmetry. Their structure is comprised of five octameric, spring-like protofibrils harboring 40 vimentin polypeptide chains in cross-section. The protofibrils are connected by the intrinsically disordered head and helix $1 \mathrm{~A}$ domains of vimentin. Individual filaments display two polymerization states characterized by either the presence or absence of a luminal density along the helical axis. The structure of vimentin filaments unveils the generic building plan of the intermediate filament superfamily in molecular details. 


\section{INTRODUCTION}

32

Vimentin is an intermediate filament (IF) protein which assembles into extensive cytoskeletal networks mainly in cells of mesenchymal origin ${ }^{1,2}$. Vimentin IFs (VIFs) participate in a broad range of cellular processes, such as the development of focal adhesions ${ }^{3}$, the protrusion of lamellipodia ${ }^{4}$, the assembly of stress-fibres ${ }^{5}$, the elongation of invadopodia ${ }^{6}$, the regulation of the dynamic properties of microtubules ${ }^{7,8}$, and even virus infection ${ }^{9,10}$. Vimentin expression and the assembly of VIFs are canonical markers and regulators of the epithelial-mesenchymal transition ${ }^{11}$ and are involved in many aspects of cancer initiation and progression ${ }^{12,13}$, and other pathophysiological conditions ${ }^{14-17}$.

More specifically, VIFs are essential components of cellular architecture with respect to the establishment and maintenance of the shape and mechanical integrity of cells ${ }^{18-20}$. Furthermore, VIFs form highly dynamic cytoplasmic meshworks that extend throughout the cell and which can rapidly respond to changes in the cellular environment. This fast and versatile remodelling of the VIFs comprising these meshworks depends both on their ability to exchange subunits along the filaments ${ }^{21,22}$, and on posttranslational modifications ${ }^{23,24}$. Oxidative and electrophilic modifications of vimentin induce dramatic alterations in VIF architecture and meshwork organization ${ }^{25,26}$.

Similar to other types of IF proteins ${ }^{27}$, vimentin contains an elongated, central rod domain composed of four coiled-coil a-helical segments, termed 1A, 1B, 2A, and 2B, respectively, that are connected by flexible linkers. In turn, the rod domain is flanked by intrinsically disordered $\mathrm{N}$-terminal head $(\mathrm{H})$ and C-terminal tail $(\mathrm{T})$ domains (Fig. 1a). Vimentin monomers assemble into parallel homodimers ${ }^{28}, \sim 49 \mathrm{~nm}$ in length ${ }^{29}$. Snapshots into vimentin assembly in-vitro suggested that two dimers interact to form an antiparallel tetramer ${ }^{30}$, with a length of $\sim 61 \mathrm{~nm}$ and a molecular mass of $\sim 214 \mathrm{kDa}{ }^{27,29}$. This tetramer is the basic building block for the subsequent assembly of VIFs ${ }^{22,31}$.

The next steps in the hierarchy of vimentin polymerization involve the lateral and longitudinal concatenation of tetramers into higher order structures that contain multiples of 4 vimentin polypeptide chains. These higher order structures assemble into protofilaments (tetramers 
attach longitudinally, 4 monomers in cross-section) ${ }^{27}$, protofibrils (8 monomers in crosssection) ${ }^{32}$, and forming unit-length filaments (ULFs) ${ }^{33-36}$, structures which are highly variable regarding their molecular mass (32, 40, or 48 monomers in cross-section). Ultimately, ULFs fuse longitudinally to form mature VIFs ${ }^{31,37,38}$.

The extensive structural polymorphism of VIFs together with their elongated, flexible building blocks and intrinsically disordered domains, impose major challenges for the determination of their 3D structure. Nevertheless, a divide and conquer X-ray crystallographic analysis of overlapping vimentin fragments have provided an atomistic model of the nearly complete rod domain ${ }^{39}$. Based on molecular dynamic simulations much of the structure of the vimentin tetramer has been determined ${ }^{29,40,41}$. Despite this progress, the structure of VIFs in their fully assembled filamentous state within cells is required for a deeper understanding of the unique properties and many functions of VIFs.

Here, we have determined the structure of in-vivo polymerized VIFs employing cryo-electron tomography (cryo-ET) and helical reconstruction procedures. Through the classification of filament segments and the assembly of class averages into filaments of substantial length, we have been able to uncover the polymorphism along the VIFs. Disentangling individual filament states, a 3D reconstruction of VIFs has been achieved providing subnanometer resolution. VIFs feature a helical assembly of 5 spring-like protofibrils built from the $1 \mathrm{~B}, 2 \mathrm{~A}$, and $2 \mathrm{~B}$ domains of vimentin and the protofibrils are linked together by the $1 \mathrm{~A}$ and $\mathrm{H}$ domains. Our analysis provides detailed insights into the architecture of VIFs and reveals the role of individual vimentin domains within the filament structure. 


\section{RESULTS}

Cryo-ET of VIFs. Mouse embryonic fibroblasts (MEFs) are widely used for investigating the cellular organization and function of VIFs. In this study we used MEFs that form a typical VIF network as revealed by 3D structured illumination microscopy (3D-SIM) imaging (Fig. 1b). The cells were cultured on electron microscopy grids and subjected to a permeabilization procedure that preserves the VIF network, while removing soluble components from the cytoplasm and the nucleus ${ }^{42}$. The VIF networks in detergent treated cells closely resemble those of intact cells (Supplementary Fig. 1a).

Using these MEF preparations, we acquired 225 cryo-tomograms in areas with varying numbers of VIFs (Fig. 1c, Supplementary Fig. 1b, Supplementary Movie 1). The VIFs exhibit a tube-like appearance with no indications of partial unravelling, suggesting a reduced heterogeneity in comparison to the results obtained with bacterial expressed vimentin assembled into VIFs in-vitro ${ }^{43}$. Using a convolutional neural network ${ }^{44}$, we identified and extracted 390,297 segments of VIFs with a box size of $65 \times 65 \times 65 \mathrm{~nm}^{3}$. These segments were projected along the direction of the electron beam and subsequently subjected to a $2 \mathrm{D}$ classification procedure ${ }^{45,46}$ (Supplementary Fig. 1c). Surprisingly, this classification revealed two distinct structural patterns in VIFs (Supplementary Fig. 2); one is helical-like and the other is rope-like in appearance. We term these VIF state-1 and VIF state-2, respectively.

Repeat distance and power spectrum analysis. VIF state-1 class averages (Fig. 1d, Supplementary Fig. 3a) reveal two tracks of a repeating pattern of elongated, low-density regions, which are displaced relative to each other, run parallel to the outer boundaries of the filament, and are accompanied by local increases of curvature and outward protrusions (Fig. 1d, yellow asterisks). The repeat distance of the pattern is $\sim 180 \AA$, as measured by autocorrelation analysis ${ }^{47}$ (Supplementary Fig. 3b). Additionally, the class averages reveal that one filament wall appears more pronounced in projection than its counterpart (Fig. 1d, blue arrowheads), which suggests an odd number of subfilamentous entities building the filaments (Supplementary Fig. 3c). 
111 Subsequently, we combined all state-1 class averages into a common power spectrum, which

112 shows helical layer lines (Fig. 1e), thereby confirming the helical symmetry of state-1 VIFs.

113 A clearly detectable layer line is positioned at $\sim 1 / 186 \AA$, which corroborates the previous 114 autocorrelation measurement. Moreover, the Bessel peak distribution along the layer line spectrum is compatible with a helical assembly that packs five building blocks in one helical pitch, which would correspond to the observed asymmetry in the class averages.

117 Consequently, on the fifth layer line, which is positioned at $\sim 1 / 37 \AA$ (Fig. 1e, blue rectangle), a 118 meridional peak can be detected.

Determining the helical symmetry of VIFs. We confirmed the helical indexing scheme by an exhaustive helical parameter search on both filament states. Therefore, we merged all vimentin segments into one stack of particles (133,780 segments of size $\left.65 \times 65 \mathrm{~nm}^{2}\right)$ and performed an initial helical 3D classification. In order to understand the basic diameter and low-resolution features of the six class averages obtained, all helical information was discarded in the first instance and the 3D class averages were fully symmetrized along their central axis. Interestingly, the class averages differ in the presence of a luminal density along their central axis (Supplementary Figs. 4a\&b). Determinations of their diameter suggest that the increases in the diameter of VIFs coincide with increases in luminal density (Supplementary Fig. 4c). The maximum diameter difference detected is $\sim 2 \mathrm{~nm}$. The outer tube diameter is $12.7 \mathrm{~nm} \pm 0.7 \mathrm{~nm}$ and the centre-to-centre distance between the tube walls is $9.4 \mathrm{~nm} \pm 0.8 \mathrm{~nm}$ with a tube wall

131 thickness of $3.3 \mathrm{~nm} \pm 0.4 \mathrm{~nm}$. Based on these measurements, we define VIF state- 1 as those polymers exhibiting a pronounced luminal density and VIF state-2 missing the distinct mass 133 along their central axis.

134 In the following, we examined whether the two polymer states are similar with respect to their helical symmetry, so that their primary structural difference would be caused by the luminal

136 density. Therefore, we used the particle sets of the six class averages from before as separate 137 inputs for a series of unbiased, helical 3D refinements ${ }^{48,49}$, assuming that the true helical 138 symmetry generates optimal resolution. Thereby, we searched through a wide spectrum of 
139 helical rise values from $30 \AA$ to $69 \AA$ at a constant helical pitch of $186 \AA$. This brute-force search

140 visited a wide range of helical geometries that contain between 2.7 to 6.2 asymmetric units in

141 the helical pitch length. The results show that four classes reached optimal resolution values

142 around a helical rise of $37 \AA$ (Supplementary Fig. 4d), and the mean resolution over all classes

143 is optimal at a helical rise of $\sim 37 \AA$ (Supplementary Fig. 4e). Subsequently, we applied the

144 helical symmetry obtained for 3D classification of the vimentin segments and confirmed that

145 the features of the reconstructed class averages in projection match the experimental 2D class

146 averages (Supplementary Fig. 4f).

147 The results of our analyses reveal that the helical symmetries of VIF state-1 and VIF state-2 148 are similar to a first approximation, indicating therefore the luminal density does not 149 significantly alter the helical symmetry of the surrounding filament tube. In order to assess the validity of the helical rise measurements, we assumed a closely-packed helical structure, so

151 that the helical rise could be estimated based on the helical pitch and the filament diameter ${ }^{50}$.

152 Here, a helical pitch of $186 \AA$ and a filament diameter of $12.7 \mathrm{~nm}$ results in a helical rise of

$15336.8 \AA$, and the maximum diameter difference of $\sim 2 \mathrm{~nm}$ between the two polymer states is 154 implying a variation in the helical rise between $36.1 \AA$ and $37.5 \AA$. This interval defines the theoretical difference between VIF state- 1 and VIF state- 2 in terms of the helical rise, that is compatible with the measured diameter polymorphism of VIFs (Supplementary Fig. 4c).

157 Three independent sets of measurements consistently reveal a helical pitch of $\sim 186 \AA$ and a

158 helical rise of $\sim 37 \AA$. These values are in agreement with cross-linking experiments suggesting 159 a helical rise of $35.5 \AA$ for VIFs ( $z_{a}$ value in ${ }^{51}$ ), as well as scanning transmission electron 160 microscopy mass-per-length measurements. Assuming that the vimentin tetramer is the 161 asymmetric unit of the helical assembly ${ }^{22,31}$, a mass-per-length of $56 \mathrm{kDa} / \mathrm{nm}$ (third peak in 162 Fig. 9 a in ${ }^{33}$ ) directly ${ }^{52}$ yields a helical rise of $38.3 \AA$.

164 Long-range tracing of VIFs. Resolving the structural fluctuations along the VIFs requires the 165 reconstruction of extended polymer stretches ${ }^{45,53}$. Therefore, we extracted smaller segments $166\left(38 \times 38 \mathrm{~nm}^{2}\right)$ and decreased the distance separating them, in order to increase the number of 
samples to more accurately track the natural bending of the VIFs. After several rounds of 2D classification, the initially selected $\sim 1.1$ million particles were concentrated to 615,106 segments and grouped into 100 class averages (Supplementary Fig. 5). These class averages were then transformed, so that their position and orientation matched the corresponding raw segments, and by this means the VIFs were assembled from the class averages. As a result,

172 their signal-to-noise ratio is greatly improved compared to the raw images of VIFs. An example 173 of an assembled VIF is shown in Fig. 2a. The change in structural patterns along a single VIF 174 can be observed over a substantial length. To complete the long-range tracing of VIFs, we applied a straightening procedure to unbend the filaments ${ }^{1,54}$.

176 In total, we assembled 5205 VIFs of different lengths (Fig. 2b). We selected a subgroup of 389 177 of these VIFs with a length of $\geq 353 \mathrm{~nm}$. These were boxed to an equal length (1024 pixel) and we measured the repeat distance along these VIFs employing autocorrelation (Fig. 2c). The

179 result showed a long-range repeat distance of $186.5 \AA \pm 26.0 \AA$, supporting the previous measurements on single class averages.

181 Next, we calculated a combined power spectrum of these VIFs (Fig. 2d). Due to the increased resolution, a dense spectrum of layer lines is revealed and Bessel peaks confirming the previously measured helical pitch and rise values can be clearly detected (Fig. 2d, yellow arrowheads). The inset in Fig. $2 d$ focuses on the most significant Bessel peaks above the background, in a region around the putative helical pitch. The layer line at $185.6 \AA \pm 4.9 \AA$ 186 (yellow rectangle) confirms the previous helical pitch measurements and a subtle splitting of 187 the layer lines can be observed (yellow arrowheads).

These results support the notion that the helical pitch of VIFs is determined by a characteristic, constant length within the vimentin tetramer. Indeed, it was shown based on molecular dynamics simulation ${ }^{29}$ and cross-linking experiments ${ }^{55}$ that the lateral stagger of the tetramer in the $A_{11}$ binding mode (characterized by largely overlapping $1 \mathrm{~B}$ domains) is similar to the 192 helical pitch length which we have determined here. However, if the lateral spacing between 193 the subfilamentous entities within the filaments can be modulated, for example due to post194 translational modifications ${ }^{23}$, the number of tetramers that can be packed into the constant 
helical pitch length will vary ${ }^{56}$. This would result in fluctuations of both helical rise and filament diameter (Supplementary Fig. 4c), and ultimately creates a high density of layer lines in the power spectrum.

In order to substantiate this view of a fine-tuned molecular packing of VIFs, the atomic model of the vimentin tetramer ${ }^{29}$ was placed in one of the previously generated fully symmetric averages (Supplementary Fig. 4a), and subsequently helical symmetrisation was applied. In this way, VIF models of defined length and helical symmetry were produced, converted into electron density maps, and then simulated power spectra were computed. In turn, the similarity between the simulated power spectra and the experimentally obtained power spectrum of VIFs (Fig. 2d) was measured by cross-correlation.

Based on this procedure we simulated filament geometries with a packing of 4.5 to 5.5 vimentin tetramers into a constant helical pitch length of $185.6 \AA$. The resulting cross-correlation curve showed that the similarity between the power spectra simulations and the experimentally derived power spectrum is maximized for a packing of $4.95 \pm 0.05$ tetramers (Fig. 2 e). Consequently, the helical symmetry of VIFs in-situ is compatible with a fine-tuned molecular packing that converges to five tetramers in one helical pitch.

We also sought to determine the structural features and distribution of the two polymerization states along the VIFs. To this end, we conducted a helical 3D classification of the vimentin segments in four classes (Supplementary Fig. 6a). We assigned two classes with luminal density as VIF state-1 (324,386 particles) and two classes without this density as VIF state-2 $(290,720$ particles). Subsequently, we identified the respective segment positions along the assembled filaments, added a binary label that encodes the luminal density filling state, and calculated a score for each filament, reflecting its fraction of state-1 segments.

218 Based on this score, we calculated the two filament profiles shown in Fig. 2f. Here, we averaged the radial profiles of two sets of 300 assembled VIFs (length $\geq 22 \mathrm{~nm}$ ), either with 220 maximal or minimal luminal density score (blue and red profiles, respectively). The mean outer 221 diameter of the VIFs in projection is $10.9 \mathrm{~nm} \pm 1.2 \mathrm{~nm}$. The blue profile indicates a pronounced 222 luminal density in state-1 VIFs, whereas the red profile shows clearly that the luminal density 
223 in state-2 VIFs is reduced and their filament boundaries are more oriented towards the less

224 dense filament lumen. The different structural motifs of the two states along the VIFs are clearly detected and it appears that segments in the same state form clusters along the VIFs (Fig. 2g, blue and red dots, respectively).

Reconstruction of VIFs at subnanometer resolution. In order to determine the 3D structure of VIFs, we selected a class of 58,952 vimentin state- 1 segments (Supplementary Figs. 6a-c) for further refinement. The helical parameters of the selected segments were validated by an exhaustive search (Supplementary Figs. 6d\&e) and used as starting values for a 3D refinement procedure employing a local helical symmetry search using the RELION helical toolbox ${ }^{48}$. The refinement procedure converged to a helical rise of $h_{f}=37.1 \AA$ and a helical twist of $\mathrm{t}_{\mathrm{f}}=71.9^{\circ}$, which translates into $\mathrm{n}=5.0$ asymmetric units packed into a helical pitch of $185.8 \AA$. The corresponding layer lines are annotated in Fig. 2d (yellow arrowheads).

The 3D structure of state-1 VIFs was resolved to $9.6 \AA$ (Supplementary Fig. 7). The reconstruction is shown as a whole in Fig. 3a. A cut open view is presented in Fig. 3b containing the luminal density as it proceeds along the central axis of the filament. In cross-section the luminal density appears elliptical, with one axis of $3.7 \mathrm{~nm} \pm 0.3 \mathrm{~nm}$ and the second axis of $3.1 \mathrm{~nm} \pm 0.3 \mathrm{~nm}$. Furthermore, the luminal density is oriented towards identical positions along the filament tube in steps of the helical pitch (Fig. 3b, squares).

242 Our analysis shows that in-situ polymerized VIFs are tube-like structures formed by 5 units, 243 presumably protofibrils ${ }^{32}$ composed of 2 tetramers in cross section (see below). In Fig. 3c we 244 show three regions of a protofibril. The rod region (rectangle-1) is an elongated density along 245 a protofibril that is delimited from the neighbouring protofibrils by openings in the structure. 246 Along a protofibril, the rod region transitions into the hub region (rectangle-2), which can be identified by a characteristic triplet pattern. Interestingly, laterally staggered to the rod and hub 248 regions, discrete densities that bridge between neighbouring protofibrils can be identified 249 (rectangle-3). We propose that these regions serve as glue domains responsible for 250 maintaining the structural integrity of VIFs by anchoring neighbouring protofibrils. Seen from 
251 inside of the VIF (Fig. 3d), the interactions of the protofibrils through the glue regions are

252 apparent, and in Fig. 3e the complete repeating unit of a protofibril is shown.

253 In order to determine the molecular architecture of the VIFs, we constructed a model based on

254 the measured helical symmetry and the atomic model of the vimentin tetramer ${ }^{29}$. The consistency of the VIF model with the experimentally obtained structure was validated by docking, reaching a correlation coefficient of 0.81 (Supplementary Fig. 8, Supplementary

Movie 2). The VIF model (Fig. 4, Supplementary Movie 3) reveals 8 vimentin polypeptide chains in cross-section of a protofibril and therefore 40 in cross-section of a filament ${ }^{36}$. Another direct consequence of the helical symmetry which we have obtained is the mass-per-length of the VIF model $(57.8 \mathrm{kDa} / \mathrm{nm})$, which perfectly matches the data obtained by scanning transmission electron microscopy ${ }^{33,34}$.

The model we have derived from our analyses shows that the rod region of a protofibril is assembled by interactions between the 1B and 2B domains of adjacent vimentin protein chains (Fig. 4a, rectangle-1). Further along a protofibril, the 2B domains enter the hub regions (Fig. 4a, rectangle-2), where the $2 \mathrm{~A}$ domains are concentrated and $\mathrm{T}$ domains are protruding from the surface of the VIFs, explaining the protrusions previously detected in the class averages (Fig. 1d, yellow asterisks). Furthermore, the $1 \mathrm{~A}$ domains extend from the hub regions into the space between the protofibrils. Together with the $\mathrm{H}$ domains the $1 \mathrm{~A}$ domains form the 269 glue regions (Fig. 4a, rectangle-3).

270 A cross section view of the VIF model is shown in Fig. 4b. This view highlights the essential 271 role of the $1 \mathrm{~A}$ and $\mathrm{H}$ domains for filament assembly, in agreement with the findings in previous 272 studies ${ }^{18,28,33,57}$. Clearly, the model shows that the $1 \mathrm{~A}$ and $\mathrm{H}$ domains are the links between 273 the spring-like protofibrils, as visualized by omitting the respective vimentin domains in the 274 filament model (Fig. 4c).

275 A model of the vimentin ULF 34 is shown in Fig. 4d. Interestingly, both ends of the ULF are 276 formed by the $2 \mathrm{~B}$ and T domains of the vimentin protein, suggesting that these domains play 277 a vital role in the elongation of VIFs. Indeed, a mimetic peptide that interferes with the $2 \mathrm{~B}$ 278 domain drastically alters the formation of ULFs and their longitudinal annealing, as 
demonstrated in a previous study ${ }^{28}$. Assembly of a VIF with a cross section of 40 polypeptide chains requires at least two helix-turns, i.e. 10 tetramers. An alternative ULF model constructed from 5 tetramers and an elongated VIF model are presented in Supplementary Fig. 9.

\section{DISCUSSION}

In this work we reveal the structure of VIFs. We have generated a molecular model of VIFs that maps the position and spatial relationships of the vimentin protein domains within the polymer and explains its general building principles (Supplementary Fig. 10). The precision of the VIF model largely depends on two factors, the accuracy of the measured helical symmetry and the fidelity of the vimentin tetramer model ${ }^{29,40}$. Since the measured helical symmetry $\left(h_{f}=37.1 \AA, t_{f}=71.9^{\circ}\right)$ provides a subnanometer resolved 3D reconstruction of the VIFs, and agrees with previous mass-per-length measurements ${ }^{33,34}$ and results obtained from crosslinking experiments ${ }^{51,55}$, our model permits a high precision position and spatial relationship

292 determination of the vimentin protein domains building VIFs.

The observed molecular architecture of in-situ polymerized VIFs is in excellent agreement with previously published results. These include the established roles of the highly conserved $1 \mathrm{~A}$ and $2 \mathrm{~B}$ domains in VIF assembly and elongation ${ }^{18,28,33,57}$, the contribution of the $\mathrm{H}$ domains to the lateral interactions between the protofibrils ${ }^{23}$, and the number of polypeptide chains per cross-section ${ }^{36}$. Since the VIF model predicts an alternating pattern of $A_{11}$ and $A_{12}$ tetramer

298 binding interfaces along a protofibril, the VIF model explains the previously determined cross299 links as well $27,32,55$ (Supplementary Fig. 10b).

300 However, the subnanometer structural determination of the VIFs does not reveal their 301 handedness. Since the sign of the helical twist angle is a priori undetermined and the map resolution is not sufficient to directly derive it, we set $-t_{f}$, so that the protofibrils coil around the helical axis in a left-handed manner, in agreement with previous considerations based on 304 structural hierarchy ${ }^{27}$.

305 Interestingly, cellular VIFs form a homogenous assembly with respect to the number of polypeptide chains per cross-section (40 vimentin monomers), compared to in-vitro assembled 
VIF preparations, where the distribution is more heterogenous $(32,40$, and 48 vimentin monomers) ${ }^{33,34,36}$, suggesting that the cell selectively controls the polymerization of the VIFs. Furthermore, no regions containing unravelled VIFs were detected in the tomograms, as described for VIFs polymerized in-vitro from bacterial expressed vimentin ${ }^{43}$.

Nevertheless, the VIFs polymerized within cells exhibit considerable polymorphism. Indeed, we observed that the VIFs exist in two major polymerization states, which are characterized by the presence of a luminal density in state-1 VIFs, and their absence or significant suppression in state-2 VIFs. Following the course of single VIFs demonstrate that transitions between these two states are common. As a consequence, the diameter of VIFs varies locally with a maximum difference of $\sim 2 \mathrm{~nm}$, which implies fluctuations in the helical rise between $36.1 \AA$ and $37.5 \AA$, assuming a constant helical pitch. Although this variability cannot be neglected for 3D structural determination, it has only a minor influence on the general architecture of VIFs. Therefore, we suggest that the tube-like structures of state- 1 and state-2 are similar, but the main difference is the luminal density. The molecular identity of the luminal density is still not clear, although, it is likely composed of vimentin protofilaments.

The VIF structures described in this analysis are likely to retain many, if not all of their in-vivo regulated post-translational modifications and therefore are in a near native state reflecting their in-vivo structural parameters. This ensures that we are studying the assembly and 325 structure of physiologically relevant VIFs, without the requirement for the denaturing and 326 renaturing procedures typically used in studies of VIF structure, and without the prerequisite 327 to define the conditions for the polymerization in-vitro.

328 In the future, the strategy developed for this study can be applied to reveal the structure of 329 VIFs in a variety of cellular states, and can be utilized as a starting point for the structural 330 characterization of other members of the IF superfamily of cytoskeletal proteins. Larger 331 datasets in conjunction with future developments in image processing will make it possible to 332 resolve complete IF structures at atomic resolution. 


\section{Acknowledgements}

336 This work was funded by grants from the Swiss National Science Foundation (SNSF

337 31003A_179418) and the Mäxi Foundation to O.M. We thank the Center for Microscopy and 338 Image Analysis at the University of Zurich.

339

\section{Author contributions}

341 M. E. analyzed cryo-electron microscopy data and developed the methods. M. S. W. and Y. T.

342 prepared samples and recorded cryo-electron microscopy data. S. S. prepared samples and

343 recorded light microscopy data. M. E. together with R. D. G. and O. M. conceived the research

344 and wrote the manuscript.

345

\section{Declaration of interests}

347 The authors have no conflicts of interest to declare.

349 Corresponding author

350 Correspondence to Ohad Medalia (omedalia@bioc.uzh.ch) 


\section{FIGURES}
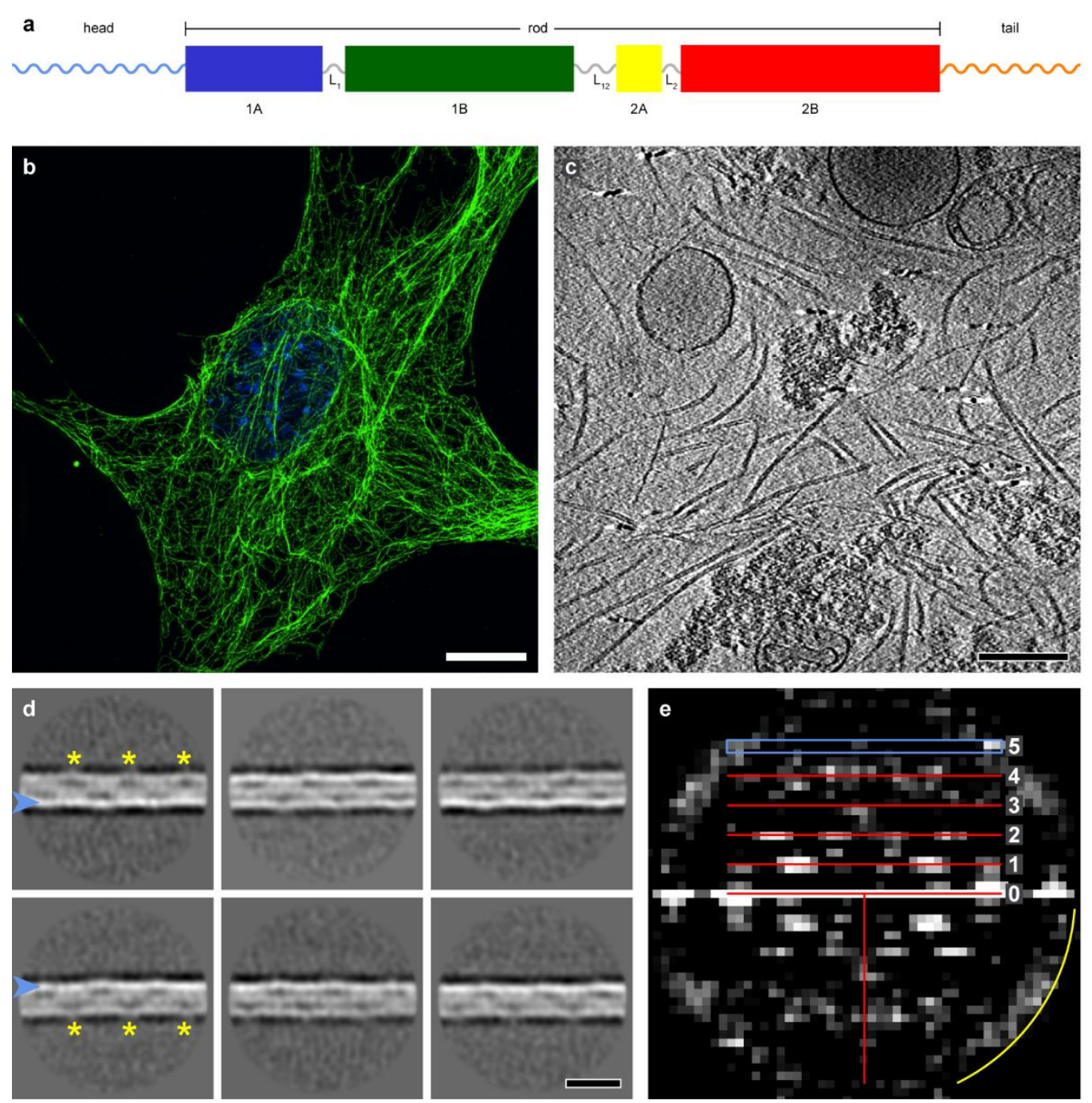

357 Figure 1. Cryo-ET and power spectrum analysis of VIFs. (a) Domain organization of a

358 vimentin monomer. The central rod consists of predominantly alpha-helical domains (1A, 1B, $3592 \mathrm{~A}, 2 \mathrm{~B})$, connected by flexible linkers ( $L 1, L 12, \mathrm{~L} 2)$. The rod is flanked by intrinsically 360 disordered head and tail domains. (b) Slice of a 3D-SIM image of a MEF, fixed and stained 361 with anti-vimentin (green) and the nucleus is stained with DAPI (blue). The VIF network 362 extends over the whole cellular volume, with regions of lower and higher network density, and 363 forms a cage around the nucleus. Scale bar is $10 \mu \mathrm{m}$. (c) Slice through a cryo-tomogram of a 364 detergent treated MEF. The VIFs can be clearly detected. They consistently show no unravelling over the whole dataset. (d) The VIF state-1 class averages show a helical pattern 
366 with a repeat distance of $\sim 180 \AA$ (yellow asterisks). One side of the filament boundary appears 367 pronounced in projection (blue arrowheads). Scale bar is $180 \AA$. (e) Combined power spectrum 368 of the VIF state-1 class averages. The presence of layer lines confirms the helical architecture. 369 The first layer line appears at $\sim 1 / 186 \AA$ and reflects the repeating pattern observed in the class 370 averages. The layer line and peak distribution with a meridional reflection on the fifth layer line 371 at $\sim 1 / 37 \AA$ is compatible with a helical assembly of five subunits per repeat. The yellow arc in 372 the power spectrum indicates $1 / 26 \AA$.

373

374

375 

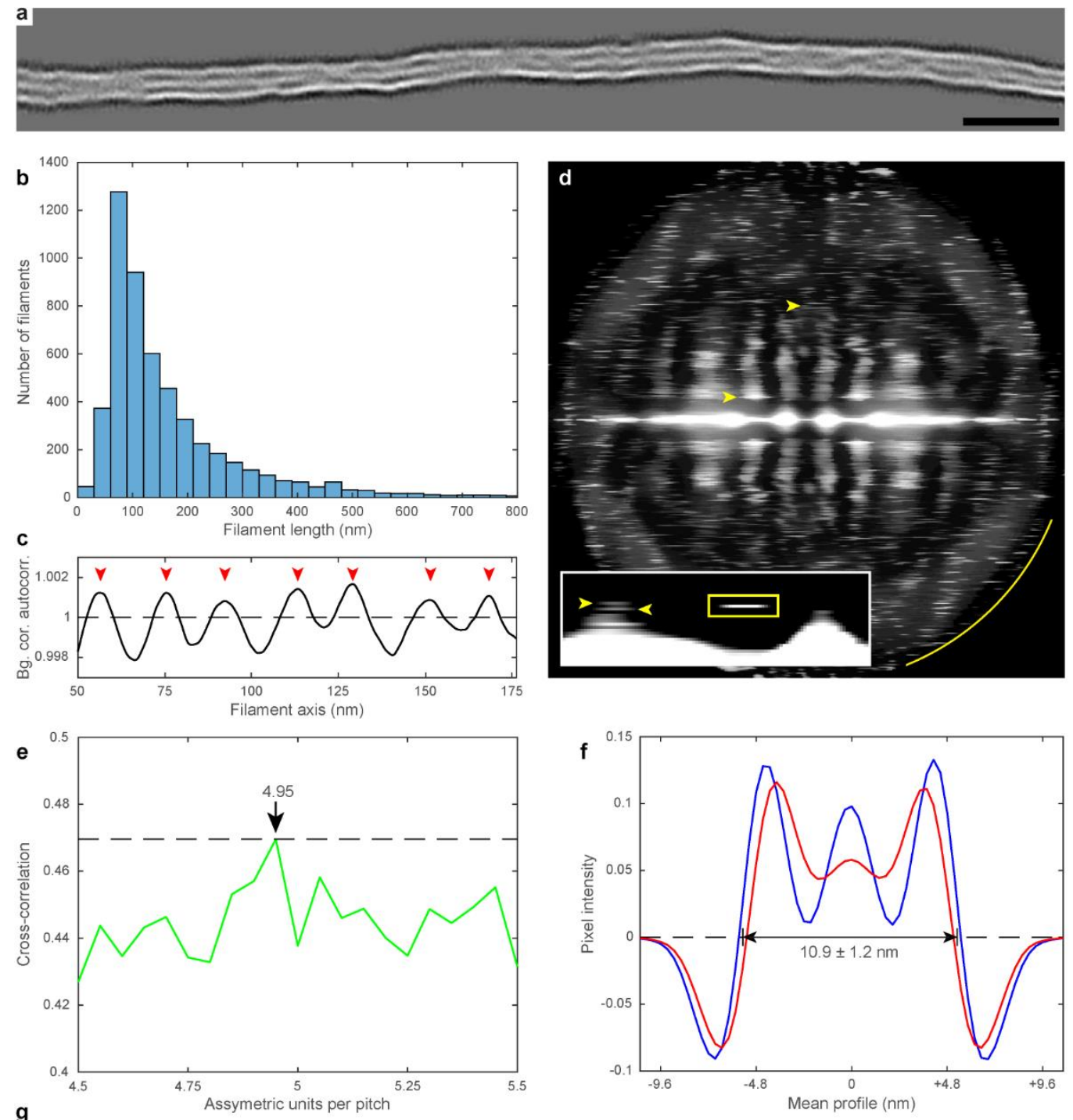

g
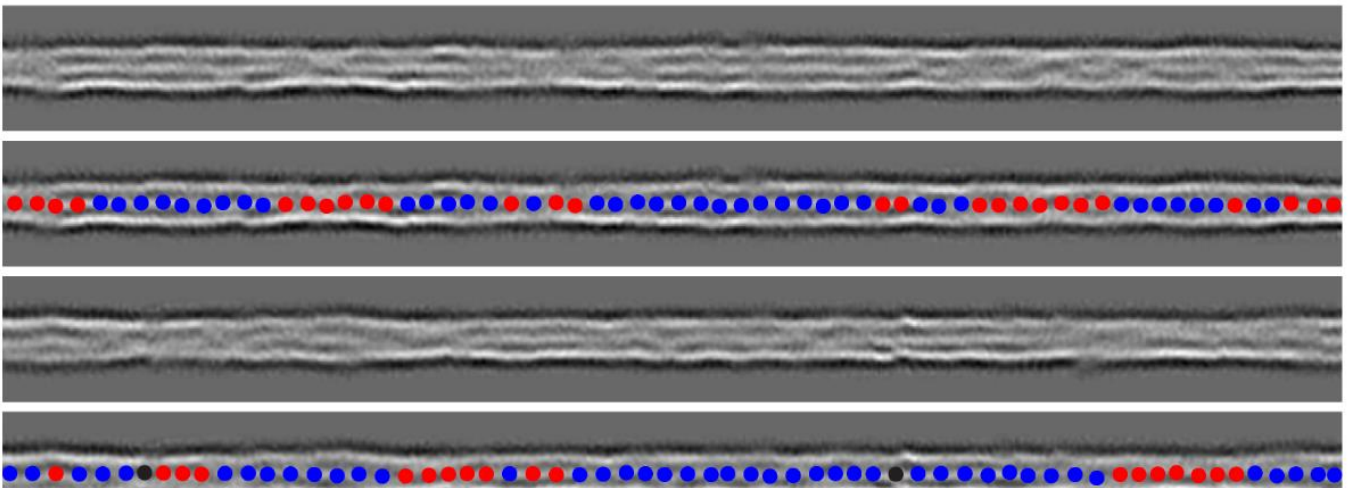

377 Figure 2. Long-range structural patterns of VIFs. (a) Filament assembly allows to follow the

378 progression of the VIFs with improved signal-to-noise ratio over a substantial length. Scale bar

379 is $35 \mathrm{~nm}$. (b) Length distribution of the assembled filaments. (c) The long-range repeat 380 distance of the VIFs was measured. The plot shows the autocorrelation signal after 381 background correction. The mean distance between the red arrowheads is $186.5 \AA \pm 26.0 \AA$. 
382 (d) The assembled VIFs were combined into one power spectrum. A dense pattern of layer 383 lines can be detected, reflecting the diffraction pattern of a discontinuous helix. The final 384 structure of state-1 VIFs converged to a helical pitch of $185.8 \AA$ and a helical rise of $37.1 \AA$. At 385 both positions sharp layer lines can be detected in the power spectrum (yellow arrowheads). 386 The yellow arc indicates $1 / 16 \AA$. In the inset at the putative pitch region a sharp layer line 387 (yellow rectangle) can be detected at $185.6 \AA \pm 4.9 \AA$. It can be observed that the layer lines 388 split into triplets (yellow arrowheads). (e) The splitting of the layer lines was modelled. At a 389 packing of $4.95 \pm 0.05$ vimentin tetramers in the length of a helical pitch the similarity between 390 the experimental power spectrum and the simulated power spectra is maximized, thereby 391 confirming the helical indexing scheme based on long-range structural patterns of VIFs. 392 (f) Two radial profiles along VIFs in state-1 and state-2 are plotted (blue and red curves, 393 respectively). In state-1 the luminal density is almost as dense as the filament walls. In state-2 394 the luminal density appears suppressed and the filament walls move inwards. (g) Two 395 assembled VIFs are shown. The blue and red dots along the filament axis indicate the position 396 of state-1 and state-2 segments, respectively. Scale bar is $35 \mathrm{~nm}$. 

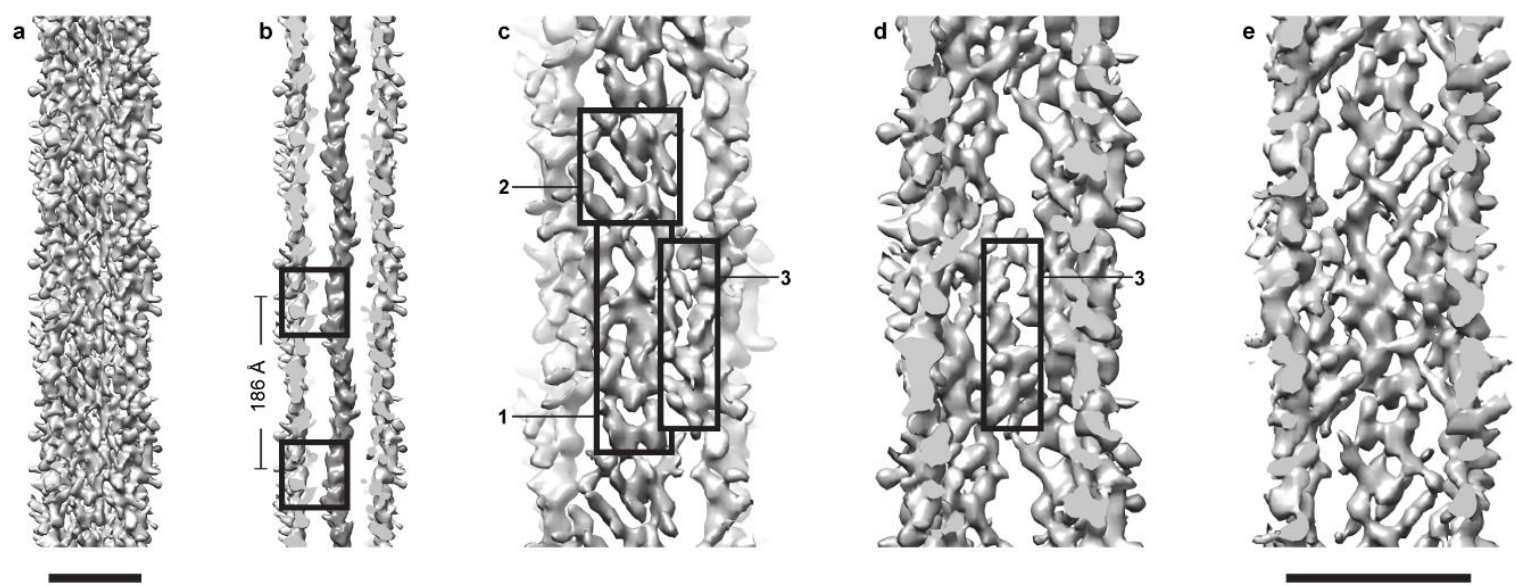

400

401

Figure 3. Helical 3D reconstruction of state-1 VIFs. (a) View of the complete structure of a

402

vimentin state-1 polymer. Scale bar is $10 \mathrm{~nm}$. (b) Cut open view of the VIF structure. The

luminal density proceeds along the central axis and is oriented towards identical positions

404

along the filament tube every $\sim 186 \AA$, as indicated by the two squares. (c) Front view of one

405

of the five protofibrils. The repeating unit can be subdivided into three characteristic regions.

406

The first region (rectangle-1) exhibits a rod like structure, that runs parallel to the filament axis.

407

The rod region is continuous with the triplet pattern of the hub region (rectangle-2). Densities

408

that connect the protofibrils (the glue regions, rectangle-3) are staggered laterally relative to

409

the rod and hub. (d) View from inside the VIF tube in one of the glue regions (rectangle-3).

410

(e) View from inside the filament tube in the repeating unit of a protofibril. Scale bar is $10 \mathrm{~nm}$. 


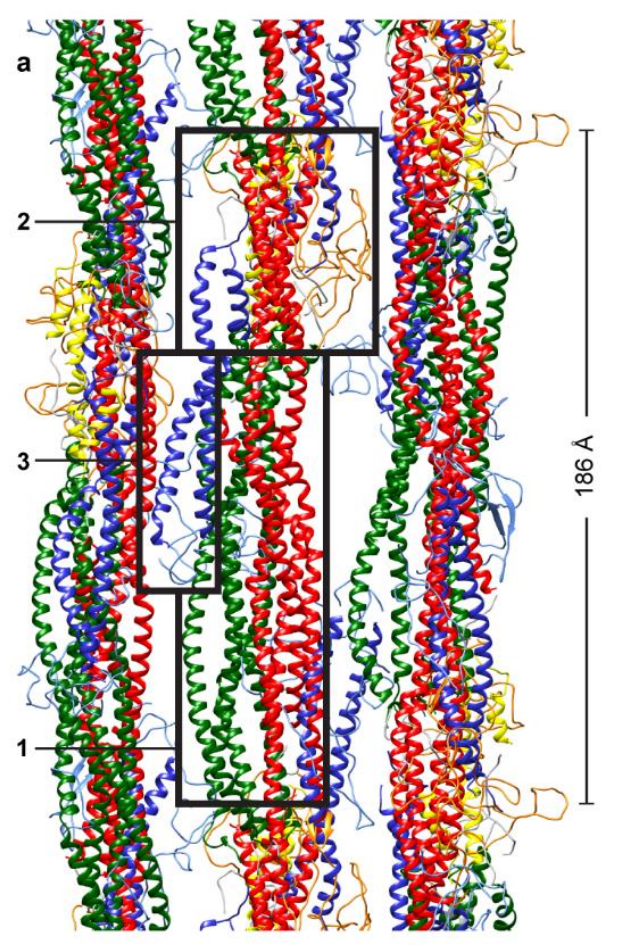

b
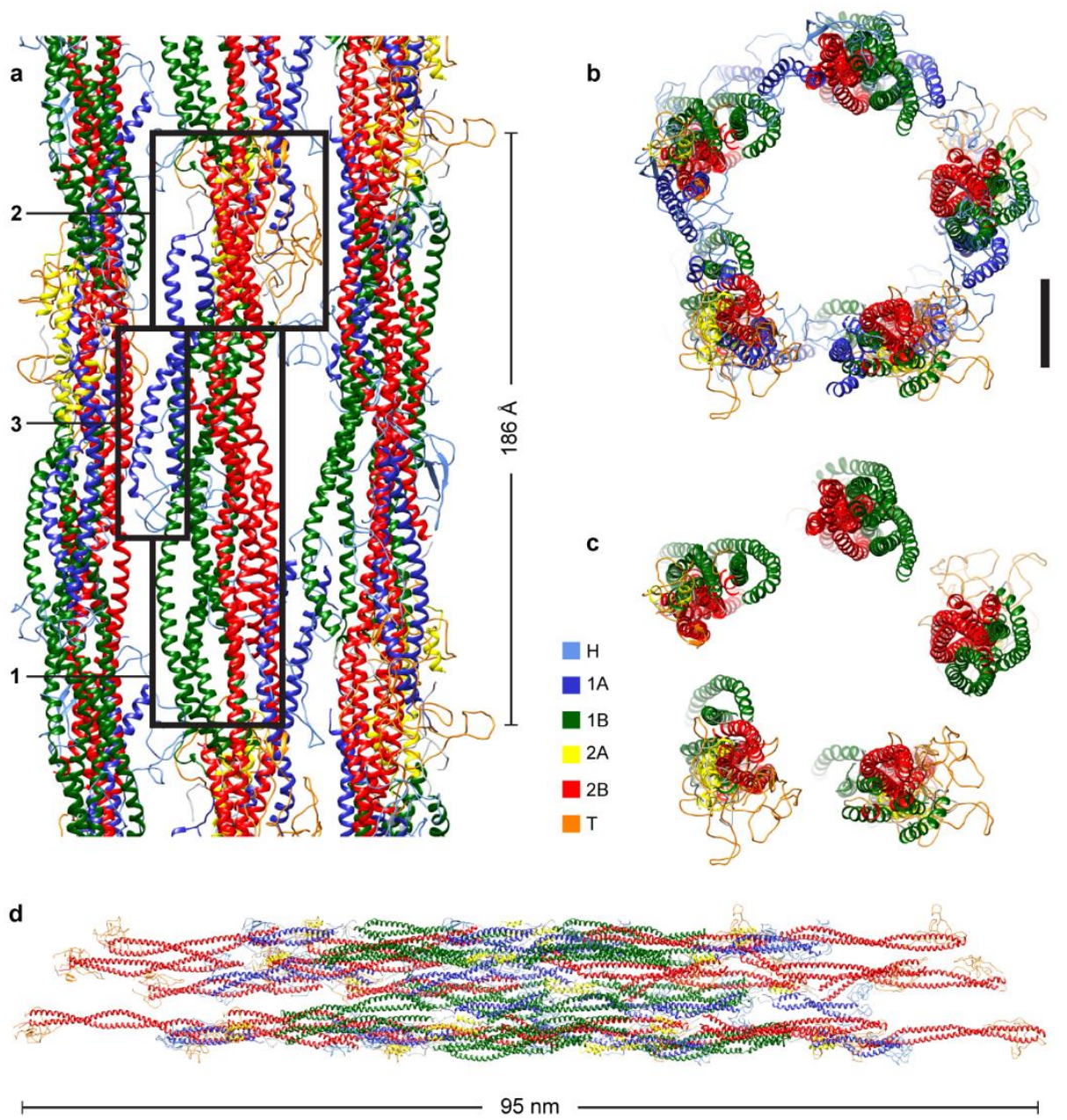

Figure 4. Molecular model of VIFs. (a) The VIF model indicates that the rod regions 416 (rectangle-1) are assembled from parallel aligned 1B and 2B domains. In the hub regions 417 (rectangle-2) the 2A domains are concentrated and the T domains are protruding away from 418 the filament surface. Furthermore, from the hub regions the $1 \mathrm{~A}$ domains extend into the space 419 between the protofibrils where they form together with the $\mathrm{H}$ domains the glue regions 420 (rectangle-3). The different protein domains are color-coded according to the color key. 421 (b) Cross-section view of the VIF model. The five protofibrils are linked together by the $1 \mathrm{~A}$ and $422 \mathrm{H}$ domains. Scale bar is $3 \mathrm{~nm}$. (c) This becomes evident by omitting the $1 \mathrm{~A}$ and $\mathrm{H}$ domains in 423 the cross-section view. (d) Molecular model of an ULF. The 2B and T domains are accessible 424 at both ends. 
428 Cell lines and cell culture. The MEFs were cultured in DMEM (Sigma-Aldrich, D5671) supplemented with 10\% FCS (Sigma-Aldrich, F7524), 2 mM L-Glutamine (Sigma-Aldrich, $\mathrm{G} 7513$ ) and $100 \mathrm{\mu g} / \mathrm{ml}$ penicillin/streptomycin (Sigma-Aldrich, $\mathrm{P} 0781$ ), at $37^{\circ} \mathrm{C}$ and $5 \% \mathrm{CO}_{2}$ in a humidified incubator.

432

433 Light microscopy. Sub-confluent cultures of MEFs growing on \#1.5 glass coverslips were 434 fixed with $4 \%$ paraformaldehyde for $10 \mathrm{~min}$ at RT. The fixed cells were permeabilized with $0.1 \%$ Triton-X 100 for $10 \mathrm{~min}$ at RT and then incubated with chicken anti-vimentin (1:200, 919101, Biolegend, CA, USA) for $30 \mathrm{~min}$ in phosphate buffered saline (PBS) containing 5\% normal goat serum. This was followed by staining with goat anti-chicken Alexa Fluor 488 (1:400, A-11039, Invitrogen, CA) and DAPI in phosphate buffered saline for $30 \mathrm{~min}$. The

439 stained cells were mounted with ProLong Glass Antifade Mountant (Life technologies, 440 Carlsbad, CA, USA).

MEFs were seeded on \#1.5 glass coverslips and the next day they were washed with PBS/2 mM MgCl 2 for $5 \mathrm{~s}$ followed by incubation with PBS containing $0.1 \%$ Triton $\mathrm{X}-100$, $10 \mathrm{mM} \mathrm{MgCl}_{2}, 0.6 \mathrm{M} \mathrm{KCl}$ and protease inhibitors for $25 \mathrm{~s}$ at $\mathrm{RT}$. The cells were rinsed with $\mathrm{PBS} / 2 \mathrm{mM} \mathrm{MgCl} 2$ for $10 \mathrm{~s}$ and subsequently incubated with 2.5 units/ $\mu$ l Benzonase for $30 \mathrm{~min}$ at RT. After rinsing with $\mathrm{PBS} / 2 \mathrm{mM} \mathrm{MgCl} 2$ the cells were fixed with $4 \%$ paraformaldehyde for

$4465 \mathrm{~min}$ at RT. The fixed cells were then stained with chicken anti-vimentin $(1: 200,919101$, 447 Biolegend, USA) and rabbit anti-lamin A (to determine the location of the nucleus) in PBS containing $5 \%$ normal goat serum for $30 \mathrm{~min}$ at RT followed by incubation with goat antichicken and anti-rabbit secondary antibodies (1:400, A-11039, A-11011, Invitrogen, USA) for

45030 min at RT. Following washing in PBS, the stained cells were mounted with Prolong Glass 451 Antifade Mountant (Life technologies, Carlsbad, CA, USA).

452 3D-SIM imaging was carried out with a N-SIM Structured Illumination Super-resolution 453 microscope system (Nikon, Tokyo, Japan) using an oil immersion objective lens 454 (SR Apo TIRF100X, 1.49 NA, Nikon). For 3D SIM, 26 optical sections were imaged at $100 \mathrm{~nm}$ 
interval. The raw SIM images were reconstructed with the N-SIM module of Nikon Elements Advanced Research with the following parameters: illumination contrast, 1.00, high-resolution noise suppression, 0.75 , and out-of-focus blur suppression, 0.25 . Brightness and contrast were adjusted for image presentation.

Cryo-ET sample preparation. MEFs were grown to $\sim 80 \%$ confluency on glow-discharged holey carbon EM grids (R2/1, Au 200 mesh; Quantifoil, Jena, Germany) prior to preparation 462 for cryo-ET analysis. Grids that showed a relatively homogenous distribution of cells were selected using fine tweezers, and then washed in PBS/2 $\mathrm{mM} \mathrm{MgCl} 2$ for $5 \mathrm{~s}$. The grids were treated for 20-40 s in pre-permeabilization buffer (PBS containing 0.1\% Triton X-100, $10 \mathrm{mM}$ $\mathrm{MgCl}_{2}, 600 \mathrm{mM} \mathrm{KCl}$ and protease inhibitors) and then rinsed in PBS/2 $\mathrm{mM} \mathrm{MgCl}$ for $10 \mathrm{~s}$. In the next step, the grids were treated with Benzonase $\left(2.5\right.$ units $/ \mu \mathrm{l}$ in $\mathrm{PBS} / 2 \mathrm{mM} \mathrm{MgCl}_{2}$; Millipore, Benzonase Nuclease HC, Purity $>99 \%$ ) for 30 min at RT. After washing the grids with PBS/2 $\mathrm{mM} \mathrm{MgCl}$, a $3 \mu \mathrm{l}$ drop of $10 \mathrm{~nm}$ fiducial gold markers (Aurion) was applied to the grids. For vitrification the grids were manually blotted for $\sim 3 \mathrm{~s}$ from the reverse side and plunge frozen in liquid ethane.

Cryo-ET. Tilt series acquisition was conducted using a Titan Krios transmission electron microscope (Thermo Fisher Scientific, Waltham, USA) equipped with a quantum energy filter

474 and a K2 Summit direct electron detector (Gatan, Pleasanton, USA). The microscope was 475 operated at $300 \mathrm{keV}$. Tilt series were collected at a nominal magnification of $42,000 \mathrm{x}$ and the 476 slit width of the energy filter was set to $20 \mathrm{eV}$. Super-resolution movies were recorded within a 477 tilt range from $-60^{\circ}$ to $+60^{\circ}$ with $2^{\circ}$ increments using SerialEM ${ }^{58}$. The image stacks were 478 acquired at a frame rate of $5 \mathrm{fps}$ with an electron flux of $\sim 2.5 \mathrm{e} / \mathrm{pixel} / \mathrm{s}$. The tilt series were 479 recorded with a total dosage of $\sim 125 \mathrm{e}^{-/} \AA^{2}$ and within a nominal defocus range between $-2 \mu \mathrm{m}$ 480 to $-6 \mu \mathrm{m}$. The super-resolution image stacks were drift-corrected and $2 \mathrm{x}$ binned using 481 MotionCorr ${ }^{59}$, resulting in a pixel size of the tilt series of $3.44 \AA$. For each tilt series image the 482 defocus was measured and the contrast transfer function (CTF) was corrected by phase- 
483 flipping. Then, from each tilt series a $4 x$ binned overview tomogram was reconstructed. The

484 CTF correction and overview tomogram reconstruction was performed using MATLAB scripts (MathWorks, Natick, USA) derived from the TOM toolbox ${ }^{60,61 .}$

486

Image processing. Algorithms from EMAN2 ${ }^{44}$ were employed for training a convolutional neural network capable of segmenting VIFs in the overview tomograms. The segmentations were manually checked and cleaned from obvious false positive VIF detections in Chimera ${ }^{62}$. Based on scripts derived from the ActinPolarityToolbox ${ }^{45}$ (APT), two sets of segment coordinates were extracted from the segmentations. In the first set (second set) the picking distance along the VIFs was set to $165 \AA$ (55 $)$, resulting in $390,297(1,148,072)$ segment coordinates. Next, based on the segment coordinates two stacks of subtomograms were reconstructed from the CTF corrected tilt series with the TOM toolbox. The dimensions of the subtomograms were $65 \times 65 \times 65 \mathrm{~nm}^{2}$ and $38 \times 38 \times 38 \mathrm{~nm}^{2}$, respectively, and the pixel size was $3.44 \AA$ A. Subsequently, APT scripts were applied to project the subtomograms, using a projection thickness of $331 \AA$ for the first set and $220 \AA$ for the second set. The size of the subtomogram projections derived from the first and second coordinate sets were $65 \times 65 \mathrm{~nm}^{2}$ and $38 \times 38 \mathrm{~nm}^{2}$, respectively. In the following, VIF segments were subjected to extensive, unsupervised 2D classifications in RELION ${ }^{46,63}$ (Supplementary Fig. 1c, Supplementary Fig. 2a, and Supplementary Fig. 5) in 502 order to sort out false positive and low signal-to-noise ratio segments. As a consequence, the 503 first particle set (second particle set) was concentrated to $133,780(615,106)$ VIF segments.

504 The Markov chain analysis (Supplementary Fig. 2d) was based on 2D classification of the first 505 particle set into 50 classes (Supplementary Fig. 2a). Employing APT scripts, the segments were connected to filaments and the sequences of the class averages along the filaments were analysed. For this purpose, a $50 \times 50$ transition matrix $(T)_{\mathrm{ij}}$ was calculated, with each matrix 508 element ij indicating the number of class average transitions from class average $\mathrm{i}$ to class 509 average $\mathrm{j}$ along the filaments. Next, $(\mathrm{T})_{\mathrm{ij}}$ was analysed with the MATLAB function $d t m c$. 
510 The assembly of VIFs (Fig. 2a and Fig. 2g) was based on the 2D classification of the second

511 particle set into 100 classes (Supplementary Fig. 5). For this purpose, the transformations

512 calculated for each segment (namely their in-plane rotation angle psi and their xy-translation)

513 were inverted and applied to the respective class averages, so that the inversely transformed

514 class averages match position and orientation of the initial segments in the image frame of the

515 tomograms ${ }^{53}$. As a result of this operation the VIFs are represented by the class averages,

516 which drastically improves their signal-to-noise ratio compared to the raw filaments.

517 Subsequently, the assembled VIFs were unbent based on a MATLAB algorithm derived from

518 the ImageJ ${ }^{64}$ straighten function ${ }^{1,54}$.

519 The autocorrelation profiles (Supplementary Fig. 3b, Fig. 2c) were calculated with the TOM 520 toolbox function tom_corr, either calculating autocorrelation functions of class averages or 521 assembled VIFs. The respective autocorrelation functions were averaged and displayed as 522 profile plots. The power spectra (Fig. 1e, Fig. 2d) were calculated with the TOM toolbox 523 function tom_ps, either calculating an averaged power spectrum from a series of class 524 averages or a series of assembled VIFs. In the second case the assembled filaments were 525 boxed to an equal length of 1024 pixel before calculating the averaged power spectrum.

526 The helical parameter searches (Supplementary Fig. 4d, Supplementary Figs. 6d\&e) utilized 527 a MATLAB script that created and executed RELION commands, and after completion 528 analysed the results. Here, the RELION function relion_refine was used extensively in 529 combination with the options -auto_refine (to ensure gold-standard resolution measurements $530{ }^{49}$ ) and -helix (to perform helical reconstruction ${ }^{48}$ ). In order to scan a certain helical parameter 531 interval, the options -helical_rise_initial and -helical_twist_initial were varied accordingly 532 between the Refine3D jobs. Subsequently, the gold-standard resolution values were plotted 533 and the helical parameter combination with the optimal resolution value was identified.

534 In order to create modelled power spectra of VIFs, the vimentin tetramer model, as obtained 535 by molecular dynamics simulation ${ }^{29,40}$, was fitted in the tube wall of one of the rotationally 536 symmetrized class averages in Chimera (Supplementary Fig. 4a). Subsequently, defined 537 helical parameter combinations were applied with the Chimera sym command to the fitted 
538 tetramer, thereby creating symmetry related tetramer copies. Next, the tetramers were 539 converted into a $10 \AA$ electron density map with the Chimera molmap command and the 540 modelled electron density maps were elongated to 1024 pixel long filament models by using 541 the RELION command relion_helix_toolbox. These structures were projected and power 542 spectra were calculated. Based on the cross-correlation coefficient (TOM toolbox function 543 tom_ccc), these modelled power spectra were compared with the experimentally measured 544 power spectrum of VIFs (Fig. 2d). Subsequently, the cross-correlation values were plotted and 545 the helical parameter combination with maximized similarity was identified (Fig. 2e).

546 Similarly, the VIF models (Fig. 4, Supplementary Fig. 9) were generated in Chimera. For this 547 purpose, the vimentin tetramer model was fitted in the tube wall of the VIF structure (Fig. 3) 548 and the measured helical symmetry $\left(h_{f}=37.1 \AA, t_{f}=71.9^{\circ}\right)$ was applied with the Chimera sym 549 command to the fitted tetramer, thereby creating a defined number of symmetry related 550 tetramer copies (10 copies in Fig. 4d and 5, 10, and 30 copies in Supplementary Fig. 9).

551 Subsequently, the tetramers were converted into an $8 \AA$ electron density map with the Chimera 552 molmap command and the modelled electron density map was docked into the VIF structure 553 with the Chimera fitmap command (Supplementary Fig. 8).

554 The VIF helical lattice visualizations (Supplementary Figs. 10a\&c) were created by 555 transforming the modelled electron density map from cartesian to cylindrical coordinates with 556 the TOM toolbox function tom_cart2cyl. This transformation was conducted for the densities 557 of each of the vimentin protein domains individually. The transformed densities were projected 558 and the resulting images were coloured and combined in one image.

560 Visualization. All isosurface representations and the visualization of the VIF model were 561 rendered with Chimera.

563 Data availability. The algorithms developed in this work are available from the corresponding 564 author upon request. The VIF structure is deposited in the Electron Microscopy Data Bank 565 under the accession code EMD-13084. 


\section{SUPPLEMENTARY MOVIES}

567

568 Supplementary Movie 1. Cryo-tomogram of a detergent treated MEF. The movie shows

569 successive slices of a representative cryo-tomogram of detergent treated MEFs. The field of 570 view of the tomogram has a size of $1.41 \times 1.41 \mu \mathrm{m}^{2}$.

571

572 Supplementary Movie 2. VIF model docked into VIF structure. The movie shows the

573 docking of the VIF model in the VIF structure (grey transparent density). The vimentin domains

574 are color-coded according to the color key. The length of the filament shown is $60 \mathrm{~nm}$.

575

576 Supplementary Movie 3. VIF model rotating around the filament axis. The vimentin

577 domains of the rotating VIF model are color-coded according to the color key. The length of 578 the modelled filament shown is $60 \mathrm{~nm}$. 

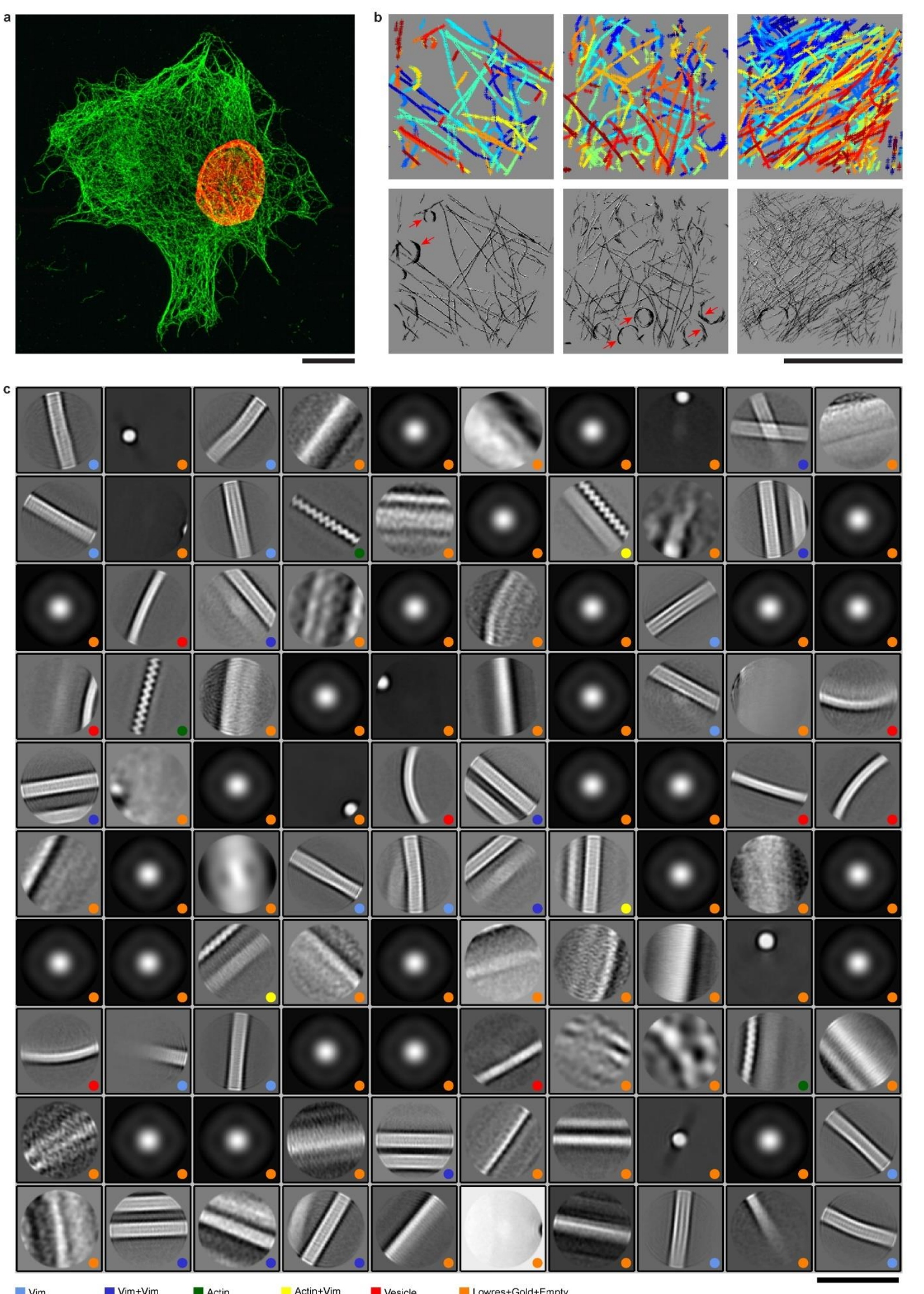

口 Vim+Vim

Vesicle Lowres+Gold+Empty 
585 Supplementary Figure 1. Deep classification of VIFs. (a) Maximum projection of a 3D-SIM 586 image of a detergent treated MEF. The VIF network remains intact following the 587 permeabilization procedure, fixation and staining with vimentin antibody (green). The cell 588 nucleus is stained in red using lamin antibody. Scale bar $10 \mu \mathrm{m}$. (b) Three examples for picking 589 VIFs with a convolutional neural network (top row). Because the filament detection is 590 performed in 3D cryo-tomograms, VIFs from densely packed regions can be analyzed as well. 591 Detected VIFs are shown in the lower panel. The convolutional neural network was over592 picking membrane structures as VIFs (red arrows). Scale bar $1 \mu \mathrm{m}$. (c) Initial 2D classification 593 of 390,297 projected vimentin segments $\left(65 \times 65 \mathrm{~nm}^{2}\right.$, picking distance $165 \AA$, projection 594 thickness $331 \AA$ A). The following patterns occur frequently in the class averages: Single VIFs 595 (classes marked with light blue dots), two VIFs running parallel or crossing on top of each other 596 (blue dots), single actin filaments (green dots), one VIF and one actin filament running parallel 597 (yellow dots), and vesicle membranes (red dots). Other categories (very low-resolution class 598 averages, gold markers or empty classes) are labelled with orange dots. Only particles from 599 single VIF classes (light blue dots) were further analyzed. Scale bar $65 \mathrm{~nm}$.

600

601

602 


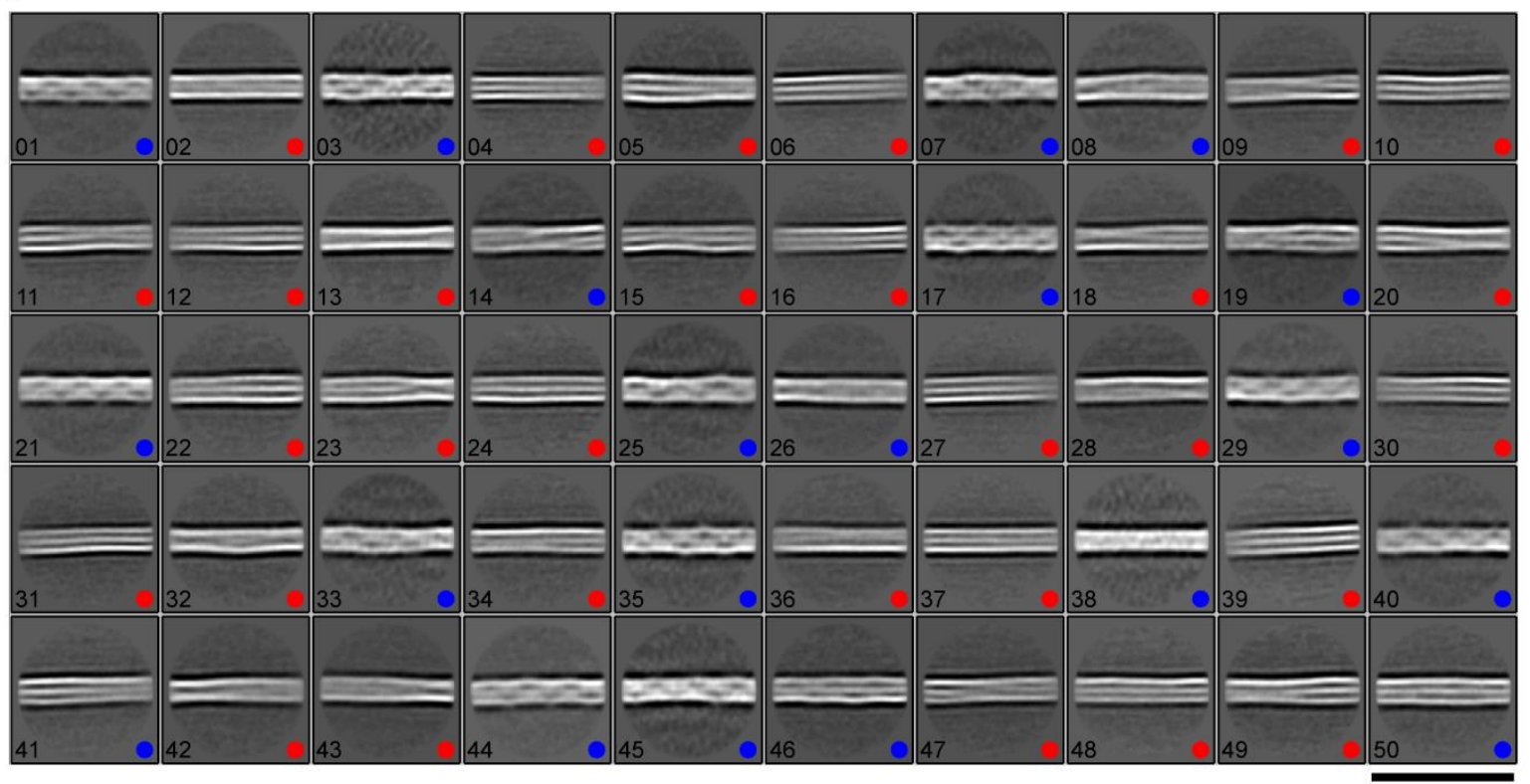

b
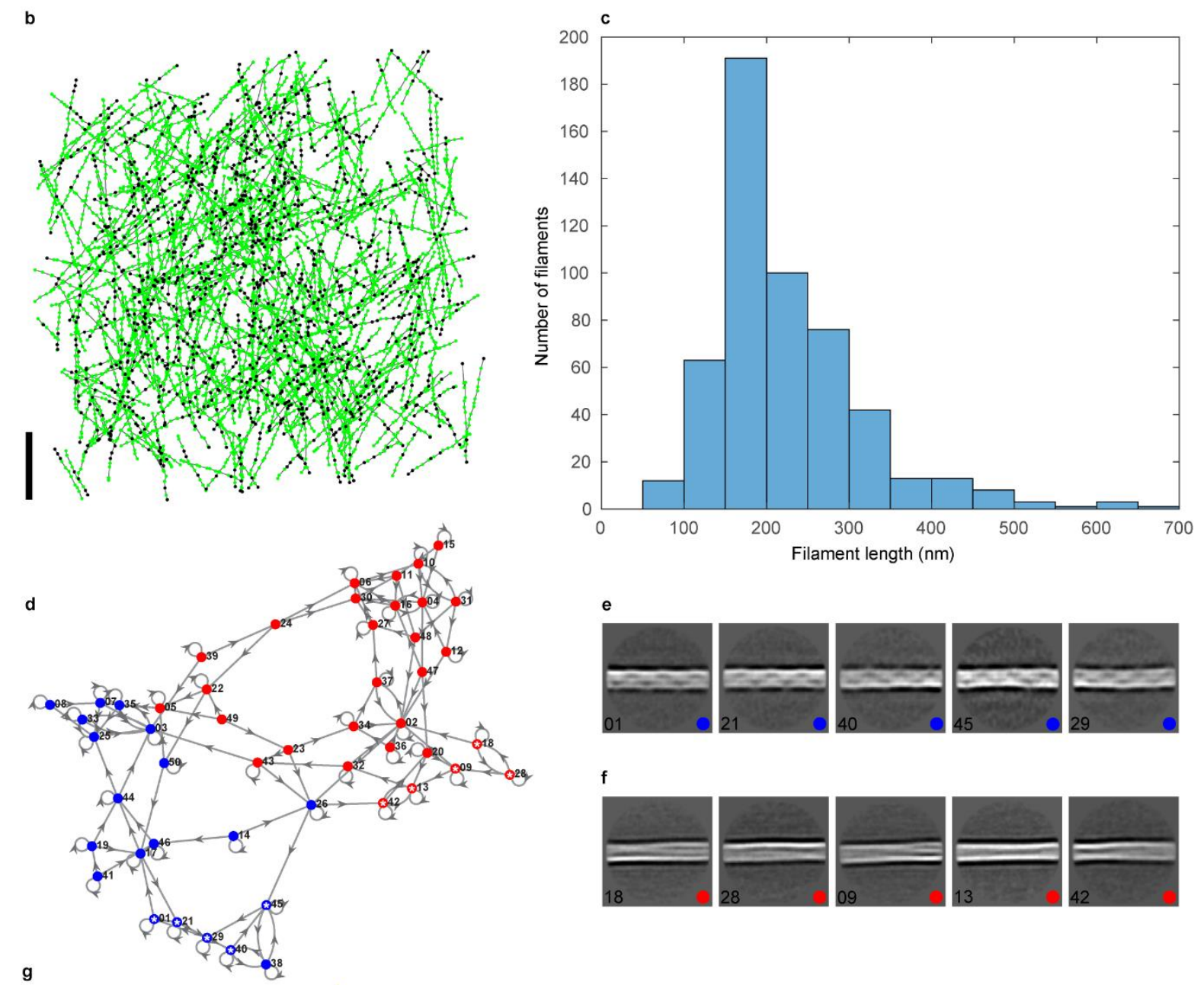

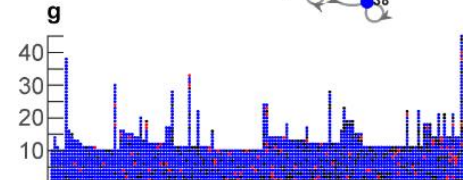


Supplementary Figure 2. Extraction and pattern analysis of VIFs. (a) Class averages of

605

606

607

608

609

610

611

612

613

614

615

616

617

618

619

620

621

622

623

624

625 626

627

628

VIFs split into two categories: The first group (VIF state-1) is characterized by similar helical patterns (class averages marked with blue dots). The second group (VIF state-2) is characterized by similar rope like patterns (class averages marked with red dots). The 2D classification is based on 133,780 segments. Scale bar $65 \mathrm{~nm}$. (b) VIFs (in total 529 with a minimum length of 10 segments) were extracted based on the 2D classification shown in (a).

Green dots represent the 3D coordinates of VIF segments, and segments plotted as black dots were sorted out during 2D classifications. Only filaments with a minimum proportion of 0.7 between processed segments (green dots) and total number of segments per filament were accepted. The orientations and positions of the filaments in the cryo-tomograms were randomly distributed. The z-coordinates of the segments were located within $\pm 90 \mathrm{~nm}$ around the central xy-plane of the cryo-tomograms. (c) Length distribution of the extracted filaments. (d) The sequences of class averages were analyzed along the VIFs with a Markov chain analysis. State-1 class averages are closely connected (blue dots), as are state-2 class averages (red dots) as well. A sequence of state-1 averages (blue dots marked with white asterisks) is shown in (e), and a sequence of state-2 averages (red dots marked with white asterisks) is shown in (f). In (g) the extracted VIFs are plotted as columns, each segment is represented by a dot, and their colors indicate the structural pattern of the segments. Blue and red dots represent state-1 and state-2 structural patterns, respectively, and black dots are segments sorted out during 2D classifications. The VIFs are sorted from left to right based on their fraction of state-1 segments. VIFs which predominantly display state-1 or state-2 patterns can be observed, as well as filaments which are transitioning between these structural states. 

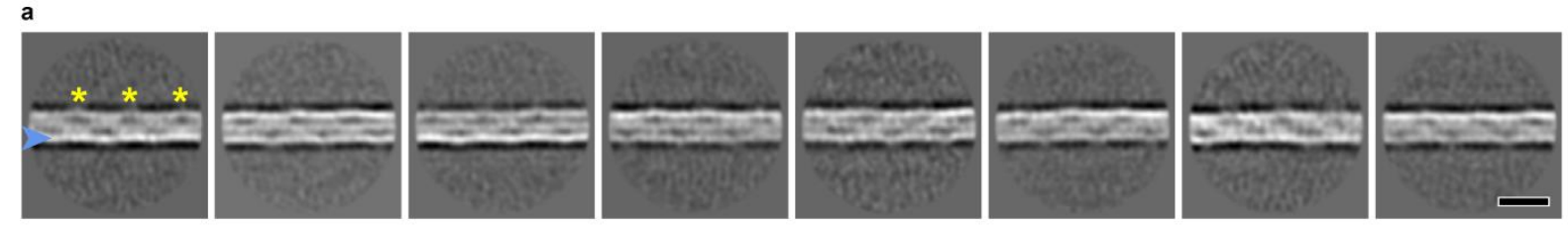

b

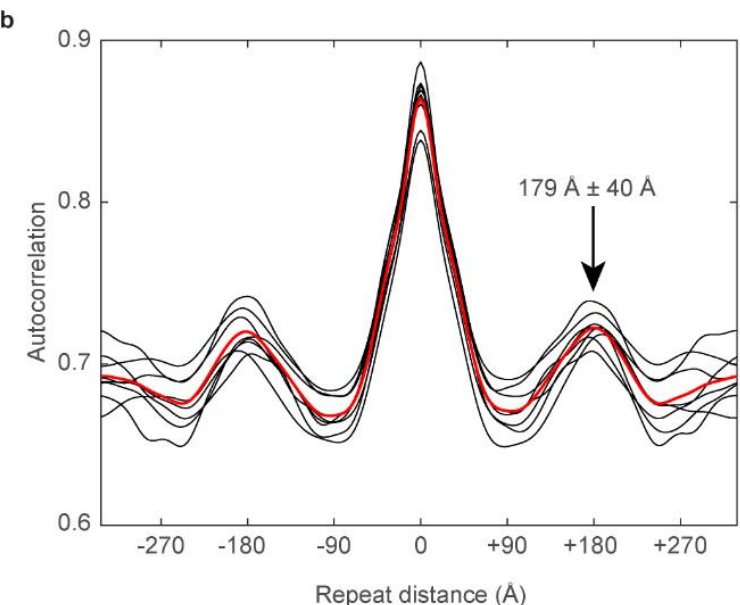

c

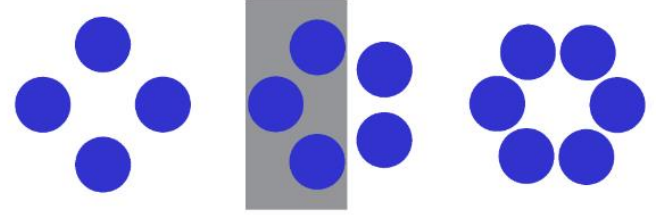

Supplementary Figure 3. Repeat distance of VIF state-1 class averages. (a) Class averages showing wall asymmetry in projection (blue arrowhead) and repeating features like elongated, parallel low-density regions together with a local increase of boundary curvature and outward protrusions (yellow asterisks). Scale bar $180 \AA$. (b) The correlation of each class average with itself was calculated and the corresponding autocorrelation profiles along the $\mathrm{x}$-axis were plotted (black lines). The red line is the averaged autocorrelation profile over all class averages. The pattern in the averages repeats at a distance of $179 \AA \pm 40 \AA$. (c) Model

637 to explain the boundary asymmetry in the class averages (blue arrowhead in (a)). If the VIFs

638 are assembled from five sub-filaments, one side of the VIF would appear brighter in projection 639 (grey rectangle). However, if they are assembled from four or six sub-filaments the VIF 640 boundaries would have similar densities in projection. 

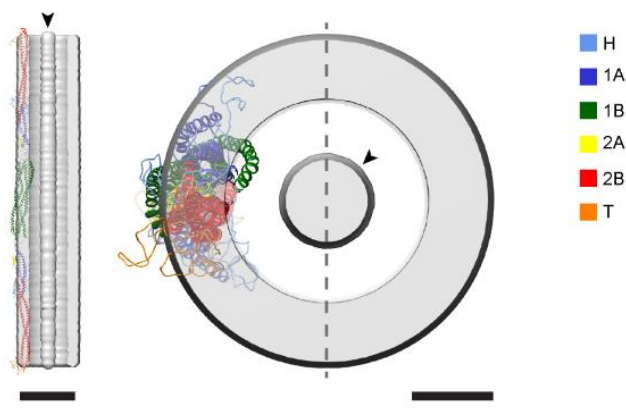

$\square 1 \mathrm{~A}$

-1B

$2 \mathrm{~A}$

$\square 2 \mathrm{~B}$

T

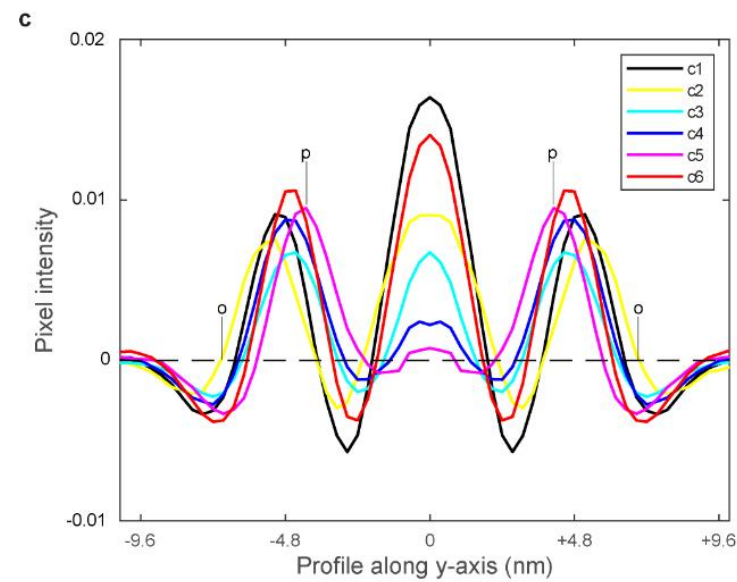

e

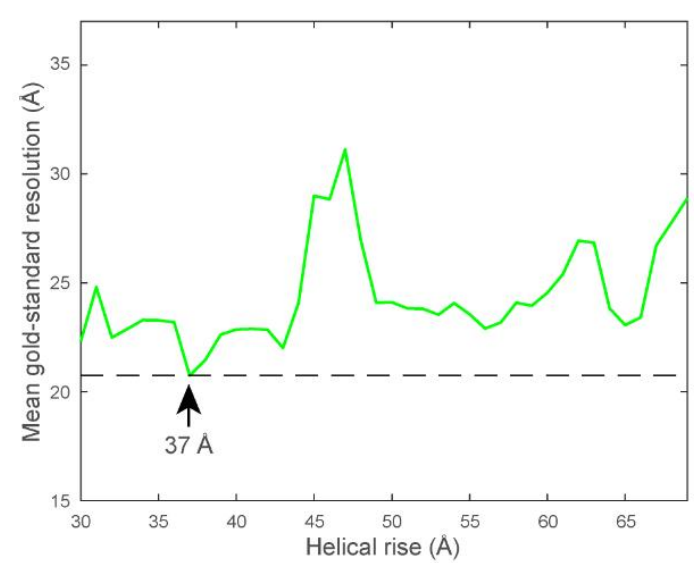

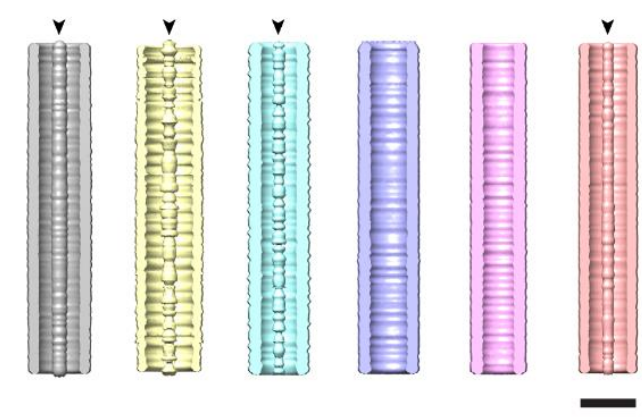

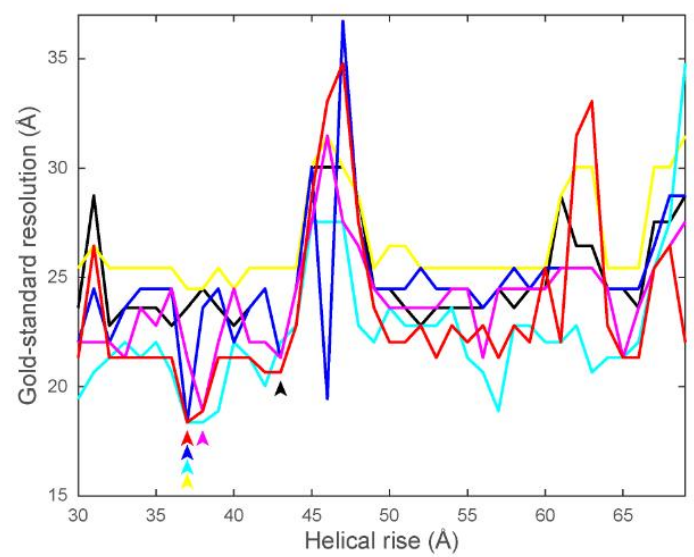

f

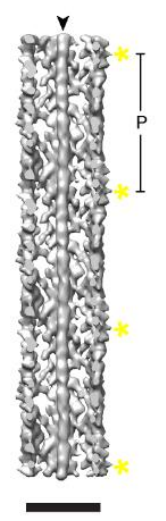

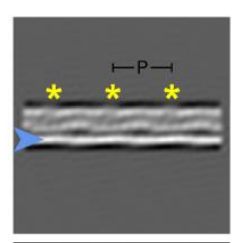
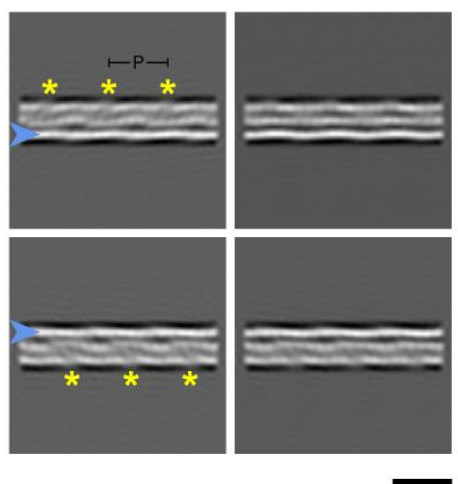

645 Supplementary Figure 4. Helical parameter search. (a) VIF segments (133,780 particles of size $65 \times 65 \mathrm{~nm}^{2}$ ) were classified in six classes and the resulting 3D averages were rotationally

647 symmetrized along their central axis. One of the tubes is shown in a cut-open view on the left 648 and shown from the top on the right. The black arrowheads indicate the luminal density. The 649 vimentin tetramer model, as obtained by molecular dynamics simulation ${ }^{29}$, has a length of $\sim 61.3 \mathrm{~nm}$ and an average diameter in cross-section of $\sim 3.6 \mathrm{~nm}$. The tetramer model was fitted

651 into the tube wall of the class average. Its tube wall thickness and curvature reflect the diameter 
and flexion of the tetramer model. The different domains of the tetramer are colored according to the color key. The vertical dashed line (y-axis) in the top view marks the path along which the profiles plotted in (c) were extracted. Left scale bar $10 \mathrm{~nm}$ and right scale bar $3 \mathrm{~nm}$. (b) The symmetrized filament tubes are shown as cut-open views. The classification splits the particle set according to filament diameter and luminal density. Prominent luminal densities are marked with black arrowheads. Scale bar $10 \mathrm{~nm}$. (c) Profile plots along the y-axis of the symmetrized class averages, referred to as c1-c6 in the legend. The average distance between the tube wall centers (p-p-distance, as exemplified for c5) is $9.4 \mathrm{~nm} \pm 0.8 \mathrm{~nm}$ and the mean outer tube diameter (o-o-distance, as exemplified for c2) is $12.7 \mathrm{~nm} \pm 0.7 \mathrm{~nm}$. The average tube wall thickness is $3.3 \mathrm{~nm} \pm 0.4 \mathrm{~nm}$. (d) For each of the particle subsets, which are corresponding to the six symmetrized 3D class averages, 40 unbiased, gold-standard 3D refinements in

663 RELION ${ }^{63}$ were calculated with constant pitch of $186 \AA$, but varying the helical rise between

$66430 \AA$ to $69 \AA$ in $1 \AA$ increments. The gold-standard resolution of the 3D refinements is plotted 665 as a function of the helical rise. The global resolution optimum for the different particle subsets 666 is indicated with arrowheads. (e) The green curve shows the average of the six resolution 667 curves displayed in (d). The global resolution optimum is located at a helical rise of $37 \AA$. 668 (f) The VIF structure shown here is the result of a helical 3D classification assuming a helical 669 pitch of $186 \AA$ and a helical rise of $37 \AA$. This class harbors a luminal density, which is marked 670 with a black arrowhead. The filament wall exhibits outward protrusions, indicated with yellow 671 asterisks, and their distance is equal to the helical pitch. Scale bar $10 \mathrm{~nm}$. In addition, four 672 reprojections of the filament structure are shown. The reprojections reproduce the features 673 observed in the experimental class averages, that are the asymmetry of the VIF boundaries in 674 projection (blue arrowheads) and parallel, low-density regions together with a local increase of 675 boundary curvature and outward protrusions (yellow asterisks). Scale bar $180 \AA$. 


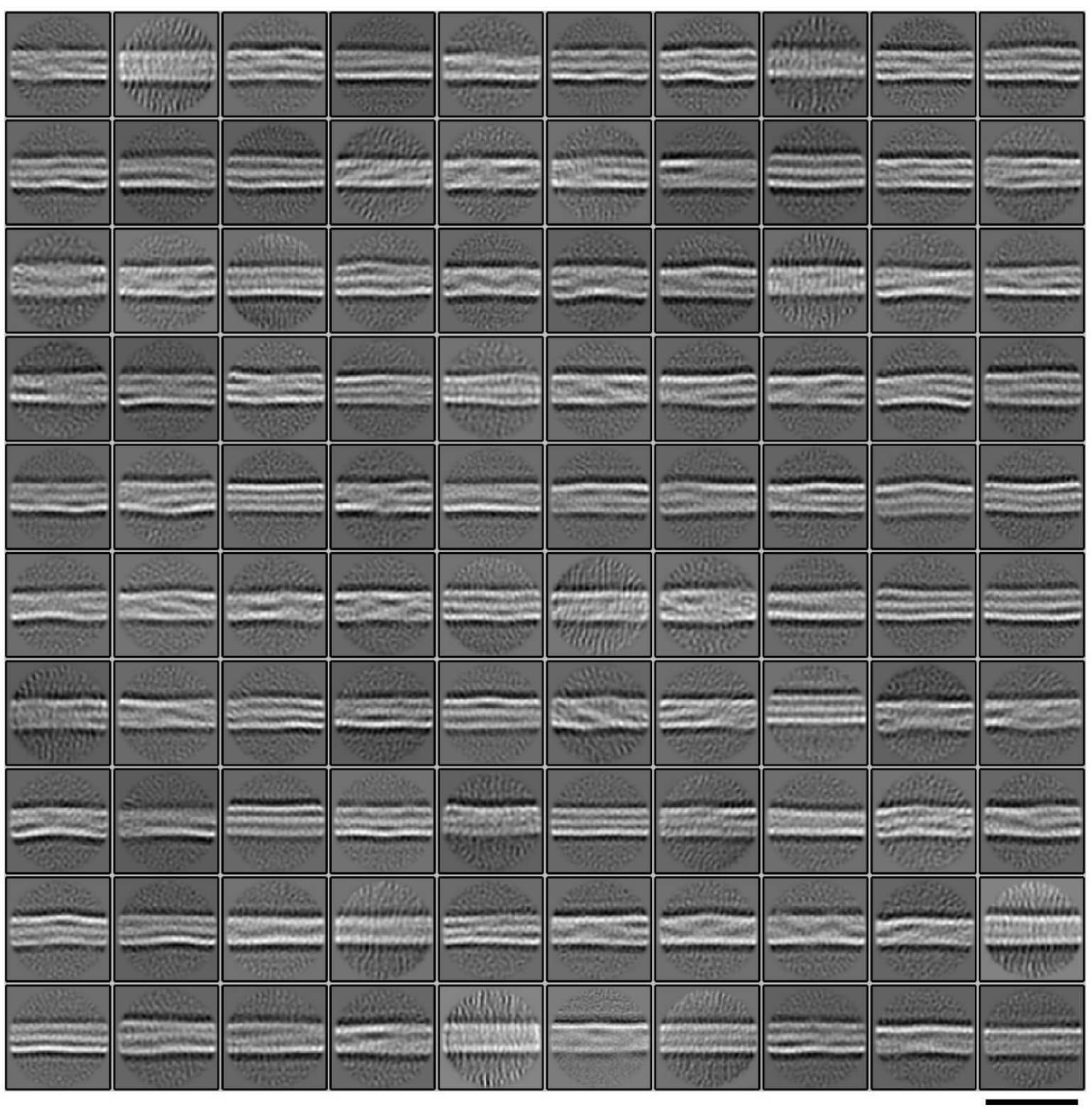

680 Supplementary Figure 5. Extended 2D classification of vimentin segments. Vimentin

681 segments $\left(615,106\right.$ particles with a size of $\left.38 \times 38 \mathrm{~nm}^{2}\right)$ were split by 2D classification in 100

682 classes. The picking distance between the segments was set to $55 \AA$ and the projection

683 thickness to $220 \AA$. The displayed class averages were used for subsequent filament 684 assembly. Scale bar $35 \mathrm{~nm}$.

685 
b
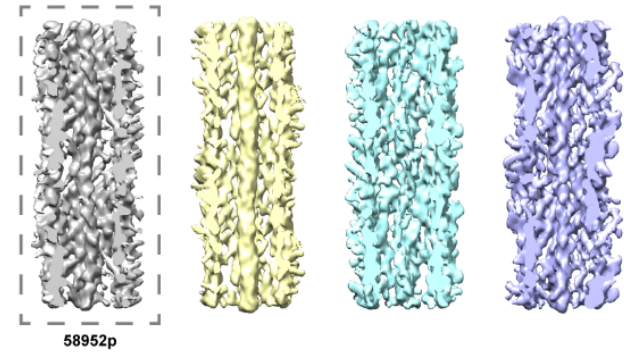

c
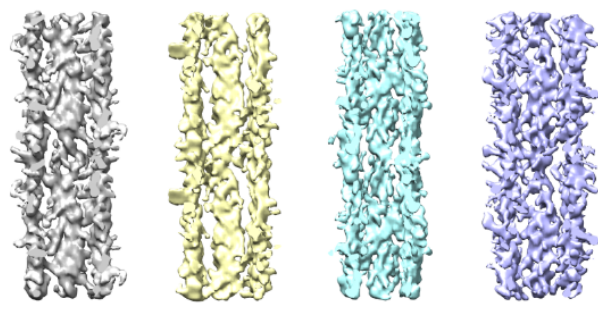

d

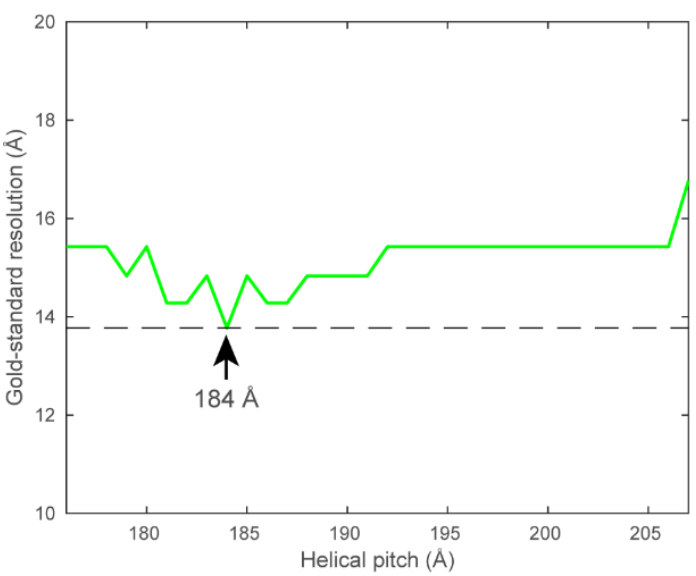

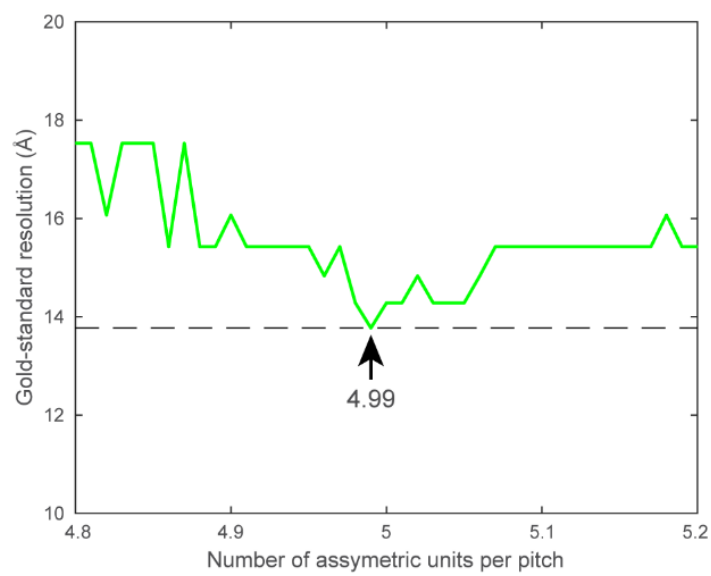

Supplementary Figure 6. Helical 3D classification of VIF segments. (a) VIF segments

$\left(615,106\right.$ particles with a size of $\left.38 \times 38 \mathrm{~nm}^{2}\right)$ were grouped by helical 3D classification in four classes (c1-c4). A helical symmetry with a helical pitch of $186 \AA$ and a helical rise of $37 \AA$ were assumed. The 3D class averages differ with respect to tube diameter and luminal density.

693 Classes c1 and c2 show pronounced luminal densities, whereas c3 and c4 show weak or no 694 luminal densities. This 3D classification defines which of the segments will be mapped as VIF state-1 (c1 and c2, in total 324,386 particles) or VIF state-2 (c3 and c4, in total 290,720 particles) on the assembled filaments (Fig. 2g). Classes c2 and c3 were further classified. The results are shown in (b) and (c), respectively. Scale bar $10 \mathrm{~nm}$. (d) Those segments assigned to $c 1$ in (b) (58,952 particles), were further analyzed by applying two more exhaustive helical 
699 parameter searches. The result of the first helical search is shown in (d). Here the number of 700 asymmetric subunits in one pitch was kept constant at $n=5.0$, but the helical pitch was varied 701 between $176 \AA$ to $207 \AA$ in $1 \AA$ increments. This effectively translates into varying the helical 702 rise $h_{r}$ and keeping the helical twist $h_{t}$ constant at $72^{\circ}$. The green curve shows the gold703 standard resolution values ${ }^{49}$, recorded with 3D Refine jobs in RELION ${ }^{48}$. The global resolution 704 optimum was found at a helical pitch of $184 \AA\left(\Rightarrow h_{r}=36.8 \AA, h_{t}=72^{\circ}\right)$. The result of the second 705 helical search is shown in (e). Here we kept the helical pitch constant at $185.6 \AA$ and varied $n$ 706 between 4.8 and 5.2. The optimal $n$ was found at 4.99 , therefore $h_{r}=37.2 \AA$ and $h_{t}=72.2^{\circ}$. These 707 helical parameters were used in the final 3D refinement as starting values for a local helical 708 symmetry search as implemented in RELION.

709

710 


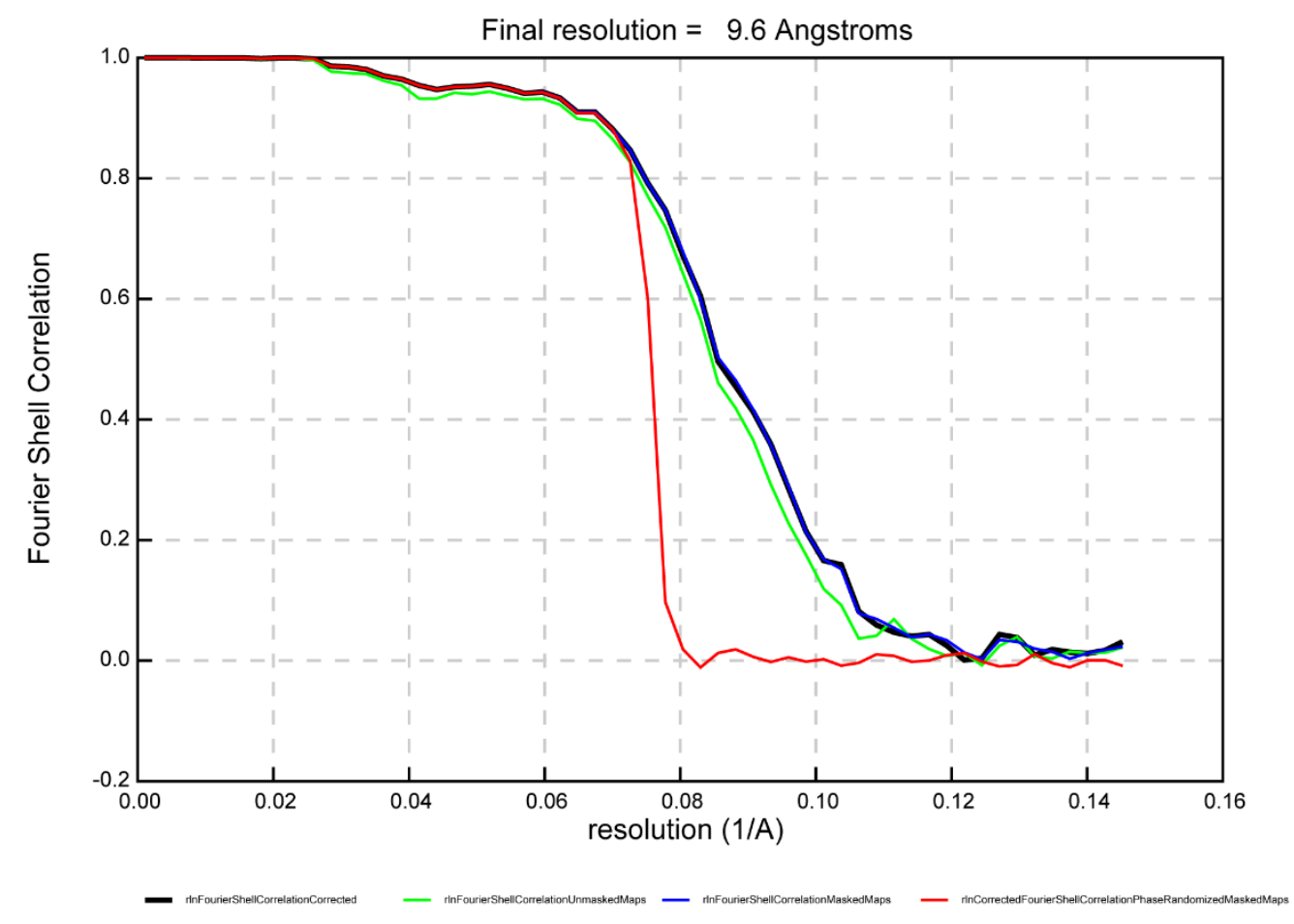

713 Supplementary Figure 7. Resolution of the resulting VIF state-1 structure. The structure 714 reached a resolution of $9.6 \AA$. 


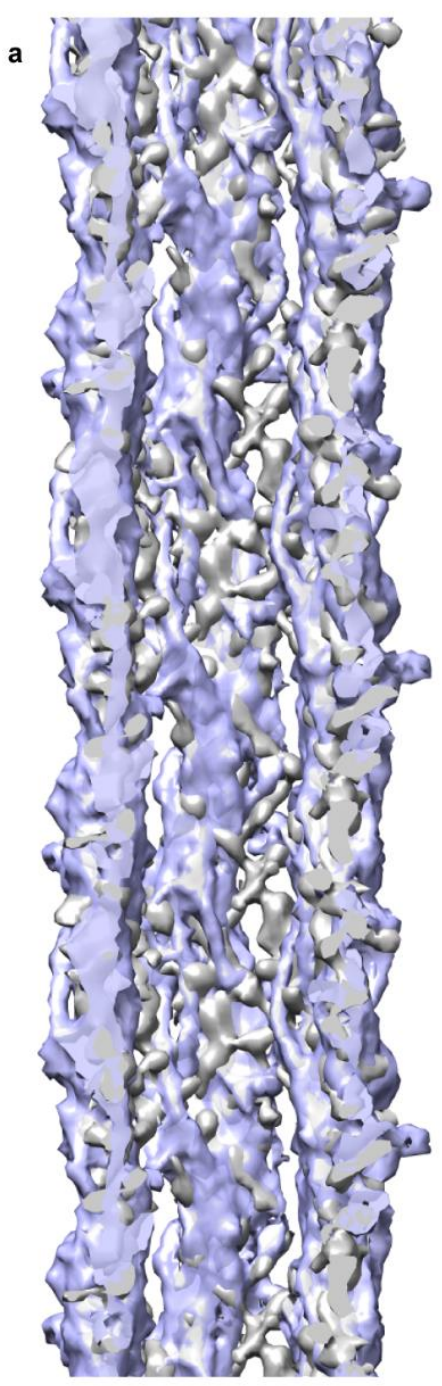

718

719 Supplementary Figure 8. Docking of the VIF model. (a) The VIF model was converted into an electron density map (blue structure) and docked into the VIF structure (grey density). The

721 correlation coefficient between the two structures is 0.81 . Scale bar $10 \mathrm{~nm}$. (b) The view 722 sections through one of the protofibrils. The overall shape of the VIF model (blue density) 723 matches the overall shape of the VIF structure (grey density). Scale bar $5 \mathrm{~nm}$.
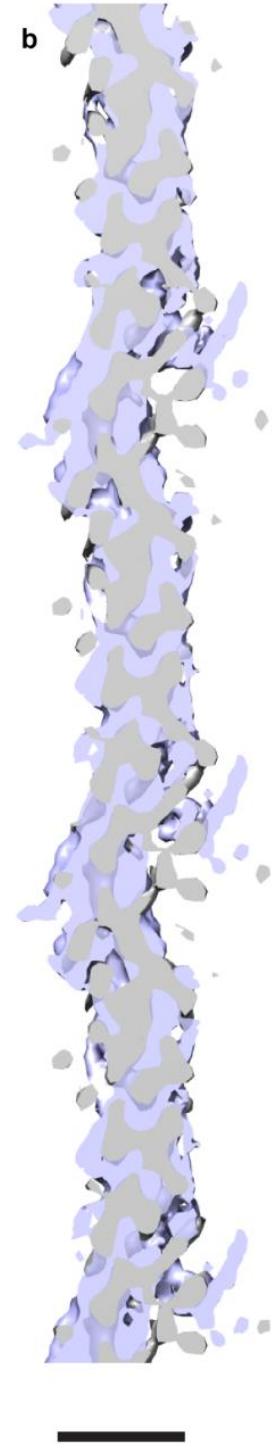
a
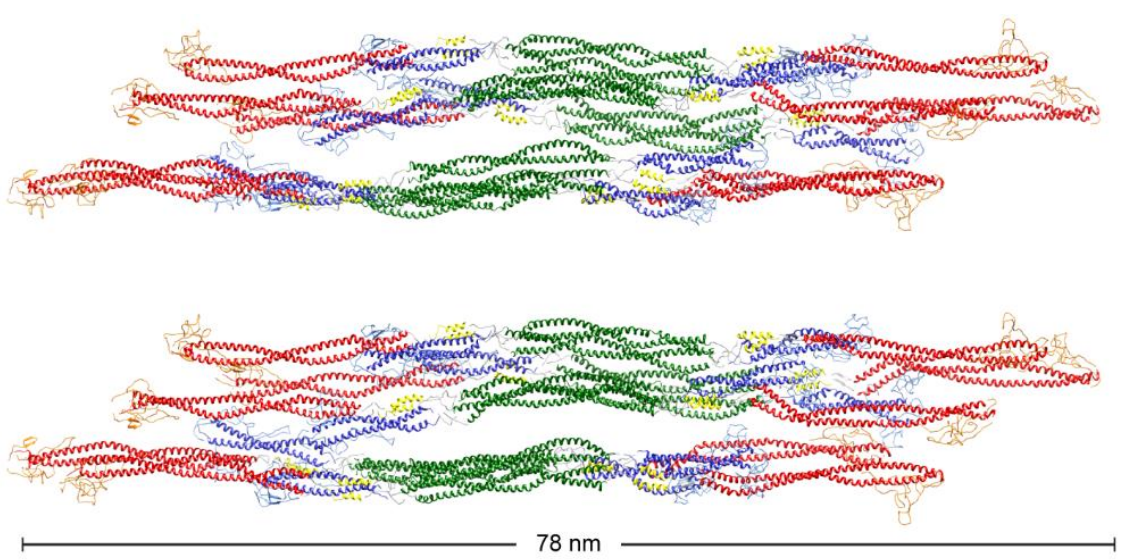

b
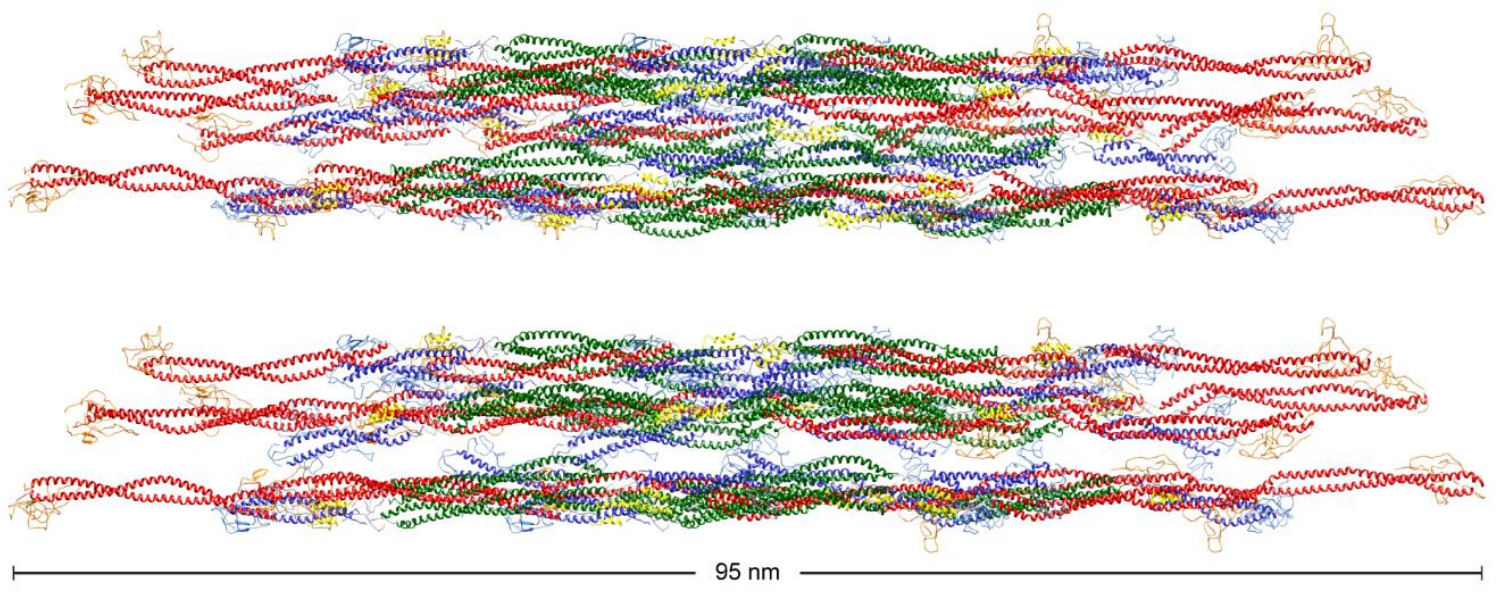

c
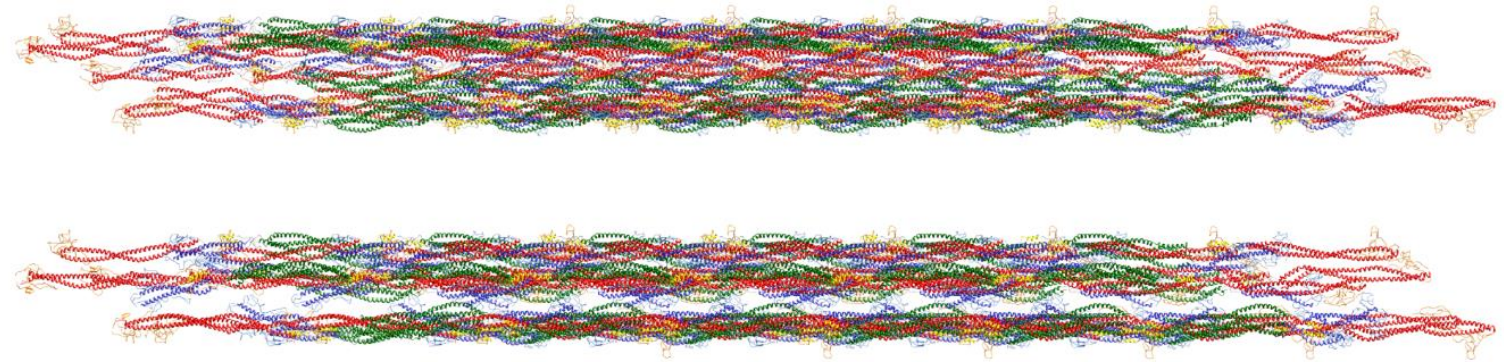

$170 \mathrm{~nm}$

728 Supplementary Figure 9. Gallery of VIF models. Three VIF models were constructed based

729 on the measured helical symmetry and the known structure of the vimentin tetramer ${ }^{29}$. The

730 models differ in the number of tetramers: (a), 5 tetramers, (b), 10 tetramers, and (c),

73130 tetramers, respectively. Each model is depicted in two views. The second view is rotated

$73218^{\circ}$ around the filament axis compared to the first view. 
733

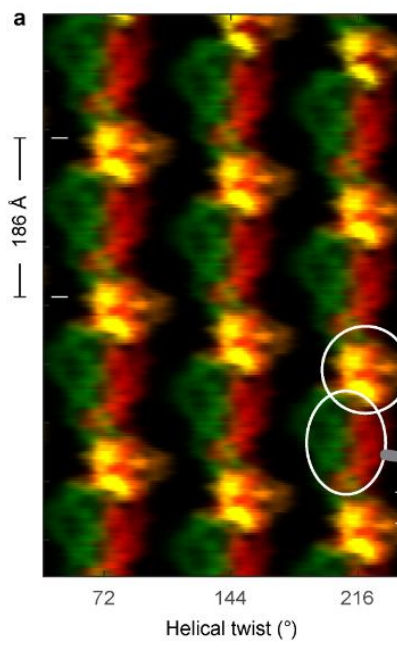

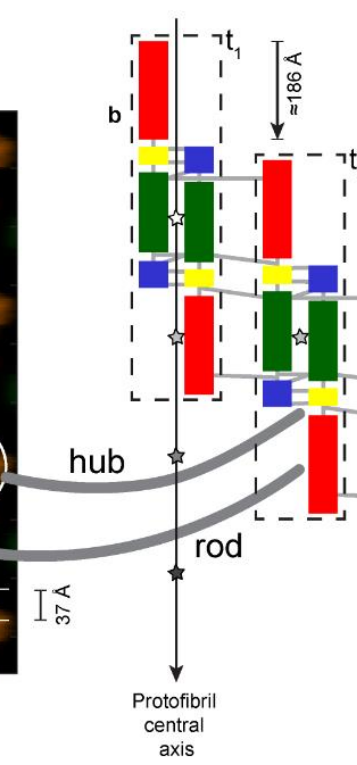
axis

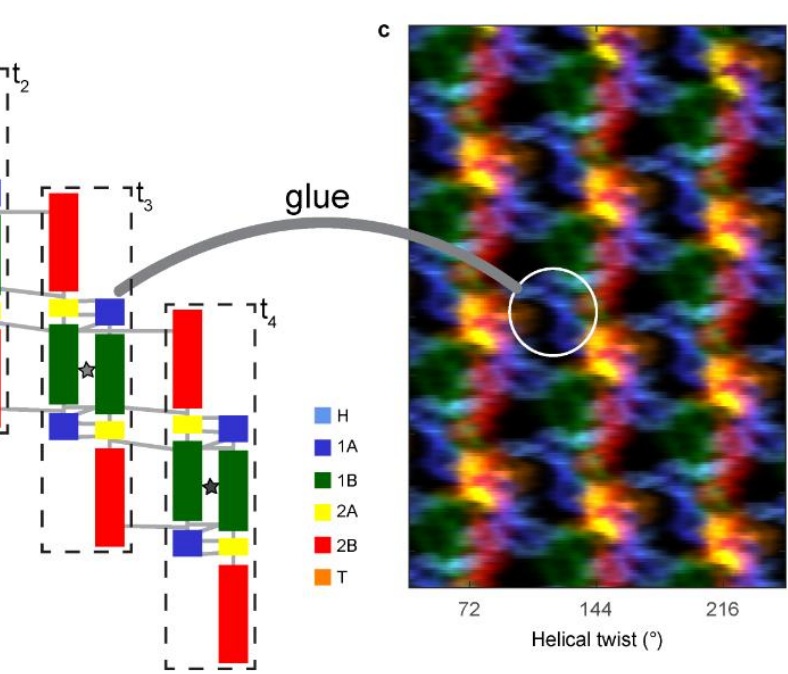

734

735

736

737

738

739

740

741

742

743

744

745

746

747

748

749

750

Supplementary Figure 10. Molecular architecture of VIFs. (a) Illustration of the helical lattice of in-situ polymerized VIFs (three of five protofibrils ${ }^{32}$ are depicted). The angular spacing between the protofibrils is $\sim 72^{\circ}$ (helical twist). Identical features along the same protofibril repeat every $\sim 186 \AA$ (helical pitch). Identical features between neighboring protofibrils are translated by $\sim 37 \AA$ (helical rise). The $1 \mathrm{~A}$ and $\mathrm{H}$ domains are omitted in this image, so there are no connections visible between the protofibrils. (b) Illustration of the domain structure of a protofibril. Four $A_{11}$ vimentin tetramers ${ }^{29,55}$ are outlined with dashed rectangles $\left(t_{1}, \ldots, t_{4}\right)$ and their centers of mass are indicated with asterisks. A protofibril is constructed by successive translations of tetramers in steps of the helical pitch. Therefore, the mass centers of the tetramers are aligned along the protofibril central axis with a distance of the helical pitch length. In the illustration, we additionally added a progressive translation of the tetramers from left to right, in order to have an unobstructed view on the individual tetramers. Since the lateral stagger between two antiparallel dimers in the $A_{11}$ vimentin tetramer is similar to the measured helical pitch, the tetramer with index $i+1$ along a protofibril creates an $A_{12}$ binding interface ${ }^{55}$ with the tetramer with index i. Therefore, our VIF model agrees with the previously determined cross-links ${ }^{55}$, which are represented by the domain connecting lines. (c) The $1 \mathrm{~A}$ and $\mathrm{H}$ domains protrude into the space between the protofibrils to form the glue regions. 


\section{REFERENCES}

7531 Steinert, P. M., Steven, A. C. \& Roop, D. R. The molecular biology of intermediate

754 filaments. Cell 42, 411-420, doi:10.1016/0092-8674(85)90098-4 (1985).

7552 Eldirany, S. A., Lomakin, I. B., Ho, M. \& Bunick, C. G. Recent insight into intermediate

756

757

758

759

760

761

762

763

764

765

766

767

768

769

770

771

772

773

774

775

776

777

778

779

780

781

782

783

784

785

786

787

788

789

790 filament structure. Current Opinion in Cell Biology 68, 132-143, doi:10.1016/j.ceb.2020.10.001 (2021).

3 Tsuruta, D. \& Jones, J. C. The vimentin cytoskeleton regulates focal contact size and adhesion of endothelial cells subjected to shear stress. J Cell Sci 116, 4977-4984, doi:10.1242/jcs.00823 (2003).

4 Helfand, B. T. et al. Vimentin organization modulates the formation of lamellipodia. Mol Biol Cell 22, 1274-1289, doi:10.1091/mbc.E10-08-0699 (2011).

5 Jiu, Y. et al. Vimentin intermediate filaments control actin stress fiber assembly through GEF-H1 and RhoA. J Cell Sci 130, 892-902, doi:10.1242/jcs.196881 (2017).

6 Schoumacher, M., Goldman, R. D., Louvard, D. \& Vignjevic, D. M. Actin, microtubules, and vimentin intermediate filaments cooperate for elongation of invadopodia. J Cell Biol 189, 541-556, doi:10.1083/jcb.200909113 (2010).

7 Gan, Z. et al. Vimentin Intermediate Filaments Template Microtubule Networks to Enhance Persistence in Cell Polarity and Directed Migration. Cell Syst 3, 252-263 e258, doi:10.1016/j.cels.2016.08.007 (2016).

8 Shabbir, S. H., Cleland, M. M., Goldman, R. D. \& Mrksich, M. Geometric control of vimentin intermediate filaments. Biomaterials 35, 1359-1366, doi:10.1016/j.biomaterials.2013.10.008 (2014).

$9 \mathrm{Yu}, \mathrm{Y}$. T. C. et al. Surface vimentin is critical for the cell entry of SARS-CoV. J Biomed Sci 23, doi:ARTN 14

10.1186/s12929-016-0234-7 (2016).

10 Suprewicz, L. et al. Vimentin binds to SARS-CoV-2 spike protein and antibodies targeting extracellular vimentin block in vitro uptake of SARS-CoV-2 virus-like particles. bioRxiv, doi:10.1101/2021.01.08.425793 (2021).

11 Mendez, M. G., Kojima, S. \& Goldman, R. D. Vimentin induces changes in cell shape, motility, and adhesion during the epithelial to mesenchymal transition. FASEB J 24, 1838-1851, doi:10.1096/fj.09-151639 (2010).

12 Kidd, M. E., Shumaker, D. K. \& Ridge, K. M. The role of vimentin intermediate filaments in the progression of lung cancer. Am J Respir Cell Mol Biol 50, 1-6, doi:10.1165/rcmb.2013-0314TR (2014).

13 Satelli, A. \& Li, S. L. Vimentin in cancer and its potential as a molecular target for cancer therapy. Cell Mol Life Sci 68, 3033-3046, doi:10.1007/s00018-011-0735-1 (2011).

791

792

793

794

795

796

797

798

14 Danielsson, F., Peterson, M. K., Caldeira Araujo, H., Lautenschlager, F. \& Gad, A. K. B. Vimentin Diversity in Health and Disease. Cells 7, doi:10.3390/cells7100147 (2018).

15 Muller, M. et al. Dominant cataract formation in association with a vimentin assembly disrupting mutation. Hum Mol Genet 18, 1052-1057, doi:10.1093/hmg/ddn440 (2009).

16 Henderson, P., Wilson, D. C., Satsangi, J. \& Stevens, C. A role for vimentin in Crohn disease. Autophagy 8, 1695-1696, doi:10.4161/auto.21690 (2012).

799

17 Fernandez-Ortega, C. et al. Identification of Vimentin as a Potential Therapeutic Target against HIV Infection. Viruses-Basel 8, doi:ARTN 98 
10.3390/v8060098 (2016).

18 Goldman, R. D., Khuon, S., Chou, Y. H., Opal, P. \& Steinert, P. M. The function of intermediate filaments in cell shape and cytoskeletal integrity. J Cell Biol 134, 971983, doi:10.1083/jcb.134.4.971 (1996).

19 Lowery, J., Kuczmarski, E. R., Herrmann, H. \& Goldman, R. D. Intermediate Filaments Play a Pivotal Role in Regulating Cell Architecture and Function. J Biol Chem 290, 17145-17153, doi:10.1074/jbc.R115.640359 (2015).

20 Janmey, P. A., Euteneuer, U., Traub, P. \& Schliwa, M. Viscoelastic properties of vimentin compared with other filamentous biopolymer networks. J Cell Biol 113, 155-160, doi:10.1083/jcb.113.1.155 (1991).

21 Vikstrom, K. L., Lim, S. S., Goldman, R. D. \& Borisy, G. G. Steady state dynamics of intermediate filament networks. J Cell Biol 118, 121-129, doi:10.1083/jcb.118.1.121 (1992).

22 Noding, B., Herrmann, H. \& Koster, S. Direct observation of subunit exchange along mature vimentin intermediate filaments. Biophys J 107, 2923-2931, doi:10.1016/j.bpj.2014.09.050 (2014).

23 Kraxner, J. et al. Post-translational modifications soften vimentin intermediate filaments. Nanoscale 13, 380-387, doi:10.1039/d0nr07322a (2021).

24 Snider, N. T. \& Omary, M. B. Post-translational modifications of intermediate filament proteins: mechanisms and functions. Nat Rev Mol Cell Biol 15, 163-177, doi:10.1038/nrm3753 (2014).

25 Monico, A., Duarte, S., Pajares, M. A. \& Perez-Sala, D. Vimentin disruption by lipoxidation and electrophiles: Role of the cysteine residue and filament dynamics. Redox Biol 23, 101098, doi:10.1016/j.redox.2019.101098 (2019).

26 Perez-Sala, D. et al. Vimentin filament organization and stress sensing depend on its single cysteine residue and zinc binding. Nat Commun 6, 7287, doi:10.1038/ncomms8287 (2015).

27 Parry, D. A. \& Steinert, P. M. Intermediate filaments: molecular architecture, assembly, dynamics and polymorphism. $Q$ Rev Biophys 32, 99-187, doi:10.1017/s0033583500003516 (1999).

28 Strelkov, S. V. et al. Conserved segments 1A and 2B of the intermediate filament dimer: their atomic structures and role in filament assembly. EMBO J 21, 12551266, doi:10.1093/emboj/21.6.1255 (2002).

29 Qin, Z., Kreplak, L. \& Buehler, M. J. Hierarchical structure controls nanomechanical properties of vimentin intermediate filaments. PLoS One 4, e7294, doi:10.1371/journal.pone.0007294 (2009).

30 Sokolova, A. V. et al. Monitoring intermediate filament assembly by small-angle $\mathrm{x}$ ray scattering reveals the molecular architecture of assembly intermediates. Proc Natl Acad Sci U S A 103, 16206-16211, doi:10.1073/pnas.0603629103 (2006).

31 Kirmse, R. et al. A quantitative kinetic model for the in vitro assembly of intermediate filaments from tetrameric vimentin. J Biol Chem 282, 18563-18572, doi:10.1074/jbc.M701063200 (2007).

32 Parry, D. A., Marekov, L. N. \& Steinert, P. M. Subfilamentous protofibril structures in fibrous proteins: cross-linking evidence for protofibrils in intermediate filaments. J Biol Chem 276, 39253-39258, doi:10.1074/jbc.M104604200 (2001).

33 Herrmann, H. et al. Structure and assembly properties of the intermediate filament protein vimentin: the role of its head, rod and tail domains. J Mol Biol 264, 933953, doi:10.1006/jmbi.1996.0688 (1996). 
853

854

855

856

857

858

859

860

861

862

863

864

865

866

867

868

869

870

871

872

873

874

875

876

877

878

879

880

881

882

883

884

885

886

887

888

889

890

891

892

893

894

34 Herrmann, H., Haner, M., Brettel, M., Ku, N. O. \& Aebi, U. Characterization of distinct early assembly units of different intermediate filament proteins. J Mol Biol 286, 1403-1420, doi:10.1006/jmbi.1999.2528 (1999).

35 Mucke, N. et al. Molecular and biophysical characterization of assembly-starter units of human vimentin. J Mol Biol 340, 97-114, doi:10.1016/j.jmb.2004.04.039 (2004).

36 Mucke, N. et al. Assembly Kinetics of Vimentin Tetramers to Unit-Length Filaments: A Stopped-Flow Study. Biophys J 114, 2408-2418, doi:10.1016/j.bpj.2018.04.032 (2018).

37 Winheim, S. et al. Deconstructing the late phase of vimentin assembly by total internal reflection fluorescence microscopy (TIRFM). PLoS One 6, e19202, doi:10.1371/journal.pone.0019202 (2011).

38 Lopez, C. G., Saldanha, O., Huber, K. \& Koster, S. Lateral association and elongation of vimentin intermediate filament proteins: A time-resolved light-scattering study. Proc Natl Acad Sci U S A 113, 11152-11157, doi:10.1073/pnas.1606372113 (2016).

39 Chernyatina, A. A., Nicolet, S., Aebi, U., Herrmann, H. \& Strelkov, S. V. Atomic structure of the vimentin central alpha-helical domain and its implications for intermediate filament assembly. Proc Natl Acad Sci U S A 109, 13620-13625, doi:10.1073/pnas.1206836109 (2012).

40 Qin, Z. \& Buehler, M. J. Structure and dynamics of human vimentin intermediate filament dimer and tetramer in explicit and implicit solvent models. J Mol Model 17, 37-48, doi:10.1007/s00894-010-0696-6 (2011).

41 Gae, D. D. et al. Completion of the Vimentin Rod Domain Structure Using Experimental Restraints: A New Tool for Exploring Intermediate Filament Assembly and Mutations. Structure 27, 1547-1560 e1544, doi:10.1016/j.str.2019.07.011 (2019).

42 Turgay, Y. et al. The molecular architecture of lamins in somatic cells. Nature 543, 261-264, doi:10.1038/nature21382 (2017).

43 Goldie, K. N. et al. Dissecting the 3-D structure of vimentin intermediate filaments by cryo-electron tomography. J Struct Biol 158, 378-385, doi:10.1016/j.jsb.2006.12.007 (2007).

44 Chen, M. et al. Convolutional neural networks for automated annotation of cellular cryo-electron tomograms. Nat Methods 14, 983-985, doi:10.1038/nmeth.4405 (2017).

45 Martins, B. et al. Unveiling the polarity of actin filaments by cryo-electron tomography. Structure, doi:10.1016/j.str.2020.12.014 (2021).

46 Bharat, T. A. M. \& Scheres, S. H. W. Resolving macromolecular structures from electron cryo-tomography data using subtomogram averaging in RELION. Nat Protoc 11, 9-20, doi:10.1038/nprot.2016.124 (2016).

47 Diaz, R., Rice, W. J. \& Stokes, D. L. Fourier-Bessel reconstruction of helical assemblies. Methods Enzymol 482, 131-165, doi:10.1016/S0076-6879(10)820051 (2010).

48 He, S. \& Scheres, S. H. W. Helical reconstruction in RELION. J Struct Biol 198, $163-$ 176, doi:10.1016/j.jsb.2017.02.003 (2017).

49 Scheres, S. H. \& Chen, S. Prevention of overfitting in cryo-EM structure determination. Nat Methods 9, 853-854, doi:10.1038/nmeth.2115 (2012). 
50 Olsen, K. \& Bohr, J. The generic geometry of helices and their close-packed structures. Theor Chem Acc 125, 207-215, doi:10.1007/s00214-009-0639-4 (2010).

51 Parry, D. A., Strelkov, S. V., Burkhard, P., Aebi, U. \& Herrmann, H. Towards a molecular description of intermediate filament structure and assembly. Exp Cell Res 313, 2204-2216, doi:10.1016/j.yexcr.2007.04.009 (2007).

52 Davies, D. B., Saenger, W., Danyluk, S. S., Federation of European Biochemical Societies. \& North Atlantic Treaty Organization. Scientific Affairs Division. Structural molecular biology : methods and applications. (Plenum Press, 1982).

53 Kronenberg-Tenga, R. et al. A lamin A/C variant causing striated muscle disease provides insights into filament organization. Journal of Cell Science 134, jcs256156, doi:10.1242/jcs.256156 (2021).

54 Kocsis, E., Trus, B. L., Steer, C. J., Bisher, M. E. \& Steven, A. C. Image Averaging of Flexible Fibrous Macromolecules - the Clathrin Triskelion Has an Elastic Proximal Segment. Journal of Structural Biology 107, 6-14, doi:Doi 10.1016/10478477(91)90025-R (1991).

55 Steinert, P. M., Marekov, L. N. \& Parry, D. A. Diversity of intermediate filament structure. Evidence that the alignment of coiled-coil molecules in vimentin is different from that in keratin intermediate filaments. J Biol Chem 268, 2491624925 (1993).

56 Sachse, C. Single-particle based helical reconstruction-how to make the most of real and Fourier space. Aims Biophysics 2, 219-244, doi:10.3934/biophy.2015.2.219 (2015).

57 Hatzfeld, M. \& Burba, M. Function of type I and type II keratin head domains: their role in dimer, tetramer and filament formation. J Cell Sci 107 ( Pt 7), 1959-1972 (1994).

58 Mastronarde, D. N. Automated electron microscope tomography using robust prediction of specimen movements. Journal of Structural Biology 152, 36-51, doi:10.1016/j.jsb.2005.07.007 (2005).

$59 \mathrm{Li}, \mathrm{X}$. M. et al. Electron counting and beam-induced motion correction enable nearatomic-resolution single-particle cryo-EM. Nature Methods 10, 584-+, doi:10.1038/nmeth.2472 (2013).

60 Nickell, S. et al. TOM software toolbox: acquisition and analysis for electron tomography. J Struct Biol 149, 227-234, doi:10.1016/j.jsb.2004.10.006 (2005).

61 Eibauer, M. et al. Unraveling the structure of membrane proteins in situ by transfer function corrected cryo-electron tomography. Journal of Structural Biology 180, 488-496, doi:10.1016/j.jsb.2012.09.008 (2012).

62 Pettersen, E. F. et al. UCSF chimera - A visualization system for exploratory research and analysis. J Comput Chem 25, 1605-1612, doi:10.1002/jcc.20084 (2004).

63 Scheres, S. H. RELION: implementation of a Bayesian approach to cryo-EM structure determination.J Struct Biol 180, 519-530, doi:10.1016/j.jsb.2012.09.006 (2012).

64 Schroeder, A. B. et al. The ImageJ ecosystem: Open-source software for image visualization, processing, and analysis. Protein Sci 30, 234-249, doi:10.1002/pro.3993 (2021). 